\title{
GYSIN TRIANGLES IN THE CATEGORY OF MOTIFS WITH MODULUS
}

\author{
KEIHO MATSUMOTO
}

\begin{abstract}
In this paper, we study a Gysin triangle in the category of motives with modulus (Theorem 1.2). We can understand this Gysin triangle as a motivic lift of the Gysin triangle of log-crystalline cohomology due to Nakkajima and Shiho. After that we compare motives with modulus and Voevodsky motives (Corollary 1.6). The corollary implies that an object in $\mathbf{M D M}^{\mathrm{eff}}$ decomposes into a $p$-torsion part and a Voevodsky motive part. We can understand the corollary as a motivic analogue of the relationship between rigid cohomology and log-crystalline cohomology.
\end{abstract}

\section{INTRODUCTION}

The Gysin triangle (see [Voe00c, Prop.3.5.4]) in Voevodsky's category of motives DM $^{\text {eff }}$ is a remarkable result which is a motivic analogue of the purity theorem of étale cohomology [AGV71, 3, XVI, Thm.3.7]. In this paper we shall prove a generalization of Voevodsky's theorem in the setting of motives of modulus pairs. Our theorem is an analogue of the Gysin triangle of (log-)crystalline cohomology (see [NS08, (2.18.8.2)]). As a corollary we give a remarkable equivalence which claims that the essential parts of a motive with modulus are the $p$-torsion part and the Voevodsky part. Our proof uses the smooth blow up formula in $\mathbf{M D M}^{\mathrm{eff}}$ (see [KS19]) and a new weighted smooth blow up formula (see Section 4).

To formulate his Gysin triangle, Voevodsky uses a smooth variety and a smooth closed subvariety. To formulate our Gysin triangle in $\underline{\mathbf{M D M}}^{\text {eff }}$ we replace the smooth variety by a modulus pair with smooth total space and a modulus whose support is a strict normal crossing divisor, and replace the closed subvariety by a prime smooth Cartier divisor which intersects the modulus properly.

Situation 1.1. Let $\bar{M}$ be a smooth scheme over a field, $M^{\infty} \subset \bar{M}$ an effective Cartier divisor, $\bar{Z} \subset \bar{M}$ a smooth integral closed subscheme not contained in $M^{\infty}$ such that the support $\left|M^{\infty}+\bar{Z}\right|$ is a strict normal crossings divisor on $\bar{M}$. Write $Z^{\infty}$ for the intersection product of $M^{\infty}$ and $\bar{Z}$.

Our main goal is the following two theorem.

Theorem 1.2. (Tame Gysin triangle) In the notation of Situation 1.1, there exsit a distinguish triangle

$$
\underline{\mathbf{M}}\left(\bar{M}, M^{\infty}+\bar{Z}\right) \rightarrow \underline{\mathbf{M}}\left(\bar{M}, M^{\infty}\right) \rightarrow \underline{\mathbf{M}}\left(\bar{Z}, Z^{\infty}\right)(1)[2] \rightarrow \underline{\mathbf{M}}\left(\bar{M}, M^{\infty}+\bar{Z}\right)[1],
$$

in $\underline{M D M}^{\mathrm{eff}}$.

Theorem 1.2 leads to the following.

Corollary 1.3 (Theorem 7.1). Let $X$ be a smooth variety over $k$ which has a compactification $\bar{X}$ such that $\bar{X}$ is smooth and $|\bar{X} \backslash X|$ is a strict normal crossings divisor on $\bar{X}$, then the unit

$$
\underline{\mathbf{M}}\left(\bar{X},|\bar{X} \backslash X|_{\mathrm{red}}\right) \rightarrow \underline{\omega}^{\mathrm{eff}}(\mathbf{M}(X))
$$

of the adjunction $\underline{\omega}_{\mathrm{eff}}: \underline{\mathbf{M D M}} \mathbf{M}^{\mathrm{eff}} \rightleftarrows \mathbf{D M}^{\mathrm{eff}}: \underline{\omega}^{\mathrm{eff}}$ is an isomorphism.

Moreover, as an application of this corollary we get the following equivalence, which philosophically has been expected since the beginning of the theory of motives with modulus.

Corollary 1.4 (Corollary 8.8). If the base field $k$ has characteristic $p \geq 2$, for any modulus pair $\left(\bar{M}, M^{\infty}\right)$ such that $\bar{M}$ is smooth and $M_{\mathrm{red}}^{\infty}$ is strict normal crossing, then there is an isomorphism in $\underline{\mathbf{M D M}^{\mathrm{eff}}(k, \mathbb{Z}[1 / p])}$

$$
\underline{\mathbf{M}}\left(\bar{M}, M^{\infty}\right)_{\mathbb{Z}[1 / p]} \simeq \underline{\mathbf{M}}\left(\bar{M}, M_{\text {red }}^{\infty}\right)_{\mathbb{Z}[1 / p]} .
$$


Definition 1.5. We define $\mathbf{M D M} \mathbf{M}^{\mathrm{eff}}$ as the smallest full triangulated subcategory of $\underline{\mathbf{M D M}}{ }^{\mathrm{eff}}$ which contains all of proper modulus pairs and is closed under small coproducts.

The category MDM $^{\text {eff }}$ is equivalent to the category in [KMSY20, Definition 3.2.4] because of [KMSY20, Threorem 3.3.1(2), Theorem 5.2.2].

Theorem 1.6 (Theorem 8.9). If the base field $k$ has characteristic $p \geq 2$, admits log resolution of singularities, and $R$ is commutative ring containing $1 / p$ then

$$
\mathbf{M D M}^{\mathrm{eff}}(k, R) \simeq \mathbf{D} \mathbf{M}^{\mathrm{eff}}(k, R) .
$$

Let us discuss the relationship between our results and other work.

1.1. Relationship to the Gysin triangle for (log-)Crystalline cohomology. First lets state the Gysin triangle for crystalline cohomology, and a comparison theorem between rigid cohomology and crystalline cohomology.

Theorem 1.7 ([NS08, Eq.2.18.8.2], [Shi02]). Let $W$ be the Witt ring of the base field, let $K$ be the fractional field of $W$, and set $S=$ Spec $W$. Consider the push forward functors $f_{-/ S}: S h(-/ S)_{\text {crys }} \rightarrow$ $S h_{\mathrm{Zar}}(S)$ from the (log-)crystalline sites of log schemes over $S$ to the Zariski site of $S$, and the structure sheaves $\mathcal{O}_{-/ S}$ on $(-/ S)_{\text {crys. }}$. In the notation of Situation 1.1, there is a long exact sequence of Zariski sheaves on $S$ :

$$
\begin{aligned}
\cdots \rightarrow R^{n-2} f_{\bar{Z} / S}\left(\mathcal{O}_{\bar{Z} / W}\right)(-1) \rightarrow R^{n} f_{\bar{M} / S}\left(\mathcal{O}_{\bar{M} / S}\right) \\
\rightarrow R^{n} f_{(\bar{M}, \bar{Z} / S)}\left(\mathcal{O}_{\overline{(M}, \bar{Z}) / S}\right) \rightarrow R^{n-1} f_{\bar{Z} / S}\left(\mathcal{O}_{\bar{Z} / S}\right)(-1) \rightarrow \cdots
\end{aligned}
$$

and we have a natural and functorial isomorphism

$$
\text { comp : } H_{\text {crys }}^{i}((\bar{M}, \bar{Z}) / W) \otimes_{W} K \simeq H_{\text {rig }}^{i}(\bar{M} \backslash \bar{Z} / K) .
$$

Expectation 1.8. We expect that there exists an exact "crystalline realization functor"

$$
\mathbb{R} \Gamma_{\text {crys }}: \operatorname{MDM}^{\mathrm{eff}}(k, W) \rightarrow D(W)
$$

satisfying

$$
\begin{gathered}
\mathbb{R} \Gamma_{\text {crys }}(\underline{\mathbf{M}}(\bar{M}, \emptyset)) \simeq \mathbb{R} \Gamma\left(S, \mathbb{R} f_{\bar{M} / S}\left(\mathcal{O}_{\bar{M} / S}\right)\right) \text { and } \\
\mathbb{R} \Gamma_{\text {crys }}(\underline{\mathbf{M}}(\bar{M}, \bar{Z})) \simeq \mathbb{R} \Gamma\left(S, \mathbb{R} f_{(\bar{M}, \bar{Z}) / S}\left(\mathcal{O}_{(\bar{M}, \bar{Z}) / S}\right)\right) .
\end{gathered}
$$

In this case, the tame Gysin triangle Theorem 1.2 would be a motivic lifting of the first claim of Theorem 1.7.

Now consider rigid cohomology. Milne-Ramachandran [MR09] construct $^{1}$ a rigid realization

$$
\mathbb{R} \Gamma_{\text {rig }}: \mathbf{D M}^{\mathrm{eff}}(k, K) \rightarrow D(K)
$$

satisfying

$$
\mathbb{R} \Gamma_{\text {rig }}(M(X))=\mathbb{R} \Gamma_{\text {rig }}(X)
$$

for $X$ smooth where the right hand side is Besser's rigid complex. By Corollary 1.6 the functor $\underline{\omega}^{\text {eff }}{ }_{K}$ : $\mathbf{D M}^{\text {eff }}(k, K) \rightarrow \underline{\mathbf{M D M}} \mathbf{M}_{\text {prop }}^{\text {eff }}(k, K)$ is an equivalence, with quasi-inverse $\underline{\omega}_{\text {eff }, K}$. Since $\underline{\omega}_{\text {eff }} \operatorname{sends} \underline{\mathbf{M}}\left(\bar{M}, M_{\text {red }}^{\infty}\right)$ to $\mathbf{M}\left(\bar{M} \backslash M^{\infty}\right)$, the second claim of Theorem 1.7 produces a natural isomorphism of functors

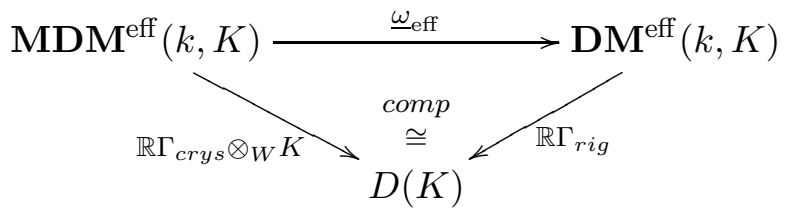

\footnotetext{
${ }^{1}$ This can be constructed as follows. Since $K$ contains $\mathbb{Q}$, by Ayoub's work [Ayo14, App.B] there is an equivalence $\mathbf{D} \mathbf{M}^{\text {eff }}(k, K) \cong \mathbf{D} \mathbf{A}_{\text {ét }}^{\text {eff }}(k, K)$ and so it suffices to construct a functor $\mathbf{D} \mathbf{A}_{\text {ét }}^{\text {eff }}(k, K) \rightarrow D(K)$. Since rigid cohomology satisfies étale descent and $\mathbb{A}^{1}$-homotopy invariance (see [CT03]), the factorization of Besser's rigid complex $\mathbb{R} \Gamma_{\text {rig }}(-):(\mathbf{S m} / k) \rightarrow$ $D(K)$ (see $[\operatorname{Bes} 00,4.9,4.13])$ through $\mathbf{D} \mathbf{A}_{\text {ét }}^{\text {eff }}(k, K)$, is a rigid realization.
} 
In this light, if Expectation 1.8 holds, then the equivalence $\underline{\omega}^{\text {eff }}$ of Corollary 1.6 will be a motivic lifting of the isomorphism comp of Theorem 1.7.

Remark 1.9. Binda-Park-Østvær constructed in [BPØ20, Section 1.3.2] a framework which is analogous to $\underline{\text { MDM }}^{\text {eff }}$ called log motives, and they are pursuing the construction of a log crystalline realisation functor in their framework. It would be very interesting to investigate the relationship between log motives and motives with modulus in the future.

1.2. Relationship to Miyazaki's works on higher Chow groups with modulus. In [BS19] BindaSaito define higher Chow groups with modulus generalizing additive higher Chow groups (see [BE03]). Miyazaki proves that after inverting $p$, higher Chow groups with modulus become independent of the modulus.

Theorem 1.10. [Miy19, Theorem 5.1] If base field has characteristic $p$, then for any modulus pair $\left(\bar{M}, M^{\infty}\right)$, we have an isomorphism

$$
\mathrm{CH}^{i}\left(\bar{M} \mid M^{\infty}, j, \mathbb{Z}[1 / p]\right) \simeq \mathrm{CH}^{i}\left(\bar{M} \mid M_{\mathrm{red}}^{\infty}, j, \mathbb{Z}[1 / p]\right) .
$$

On the other hand, it is expected that Voevodsky's isomorphism [Voe00c, Cor.4.2.9]

$$
\mathrm{CH}^{n-i}(X, j-2 i) \cong \operatorname{hom}_{\mathbf{D M}^{\mathrm{eff}}}\left(\mathbb{Z}(i)[j], M_{g m}^{c}(X)\right)
$$

can be generalized to a relationship between higher Chow groups with modulus and $\underline{\text { MDM }} \mathbf{M f f}^{\text {. If this is }}$ the case, then the equivalence of Corollary 1.6 can be seen as an analogue of Miyazaki's independence result.

1.3. Relationship to reciprocity sheaves. If the base field $k$ has characteristic $p$, then for a Nisnevich reciprocity sheaf $F$ (see [KSY14]), the kernel of the canonical surjective morphism $F \rightarrow \mathrm{H}_{0}(F)$ must be $p$ primary torsion, (see [BCKS17, Corollary 3.10]). In fact Binda-Cao-Kai-Sugiyama prove an equivalence of categories $\boldsymbol{R e c}_{\mathrm{Nis}}\left[\frac{1}{p}\right] \simeq \mathbf{H I}_{\mathrm{Nis}}\left[\frac{1}{p}\right]$ between the category of reciprocity sheaves and the category of homotopy invariant Nisnevich sheaves with transfers.

On the other hand, there is a tower of fully faithful functors $\mathbf{H I}_{\mathrm{Nis}} \stackrel{i_{\text {rec }}^{\mathrm{Nis}}}{\longrightarrow} \mathbf{R e c}_{\mathrm{Nis}} \stackrel{\omega_{\text {rec }}^{\text {CI }}}{\longrightarrow} \mathbf{C I}_{\mathrm{Nis}}^{s p}$ (see $[\mathrm{KSY} 17$, Thm.3.6.6], [KSY17, Cor.3.8.2], [BS18]). In analogy to the fact that the heart of $\mathbf{D} \mathbf{M}^{\text {eff }}$ is $\mathbf{H I}_{\mathrm{Nis}}$ (see [Voe00c, Thm.3.1.12]), it is expected that the heart of $\mathbf{M D M}{ }^{\text {eff }}$ is $\mathbf{C I}_{\mathrm{Nis}}^{\text {sp }}$ ( $\log$ version of this story is proved by Binda-Merici [BM20, Theorem 5.7]). By definition the composition $\omega_{\text {rec }}^{\text {CI }} i_{\text {rec }}^{\text {Nis }}$ is compatible with $\omega^{\text {eff }}: \mathbf{D M}^{\text {eff }} \rightarrow \mathbf{M D M} \mathbf{M}^{\text {eff }}$. If we assume that the heart of $\mathbf{M D M} \mathbf{M}^{\text {eff }}$ is $\mathbf{C I}_{\mathrm{Nis}}^{s p}$ then Cor.1.6 implies an equivalence $\mathbf{C I}_{\mathrm{Nis}}^{s p}\left[\frac{1}{p}\right] \cong \mathbf{H I}_{\mathrm{Nis}}\left[\frac{1}{p}\right]$. Then the two inclusions $i_{\mathrm{rec}}^{\mathrm{Nis}}\left[\frac{1}{p}\right]$ and $\omega_{\mathrm{rec}}^{\mathbf{C I}}\left[\frac{1}{p}\right]$ become equivalences, so Corollary 1.6 can be seen as an analogue of this story.

\section{ACKNOWLEDGEMENT}

We thank an anonymous referee for many suggestions which improved the readability of this paper.

Next, I would like to thank those members of motives with modulus school especially Federico Binda, Shane Kelly, Hiroyasu Miyazaki, and Shuji Saito.

I must also thank Jun Koizumi, a master student in Tokyo university. He pointed out a mistake in the proof of the open Gysin triangles [Koi].

\section{Definition and Preparation}

In this paper, we work over a perfect field $k$. As in [KMSY19a, Definition 1.3.1] we write $\underline{\text { MCor for }}$ the category of modulus pairs and left proper admissible correspondences. We write

$$
\mathbb{Z}_{\text {tr }}: \underline{\text { MCor }} \rightarrow P S h(\underline{\text { MCor }})
$$

for the associated representable additive presheaf functor.

We set $\underline{\text { MNST }}$ to be the category of Nisnevich sheaves on MCor defined in [KMSY19a, Definition 4.5.2]. 
We define $\underline{\mathbf{M D M}}^{\mathrm{eff}}$ to be the Verdier quotient of $\mathbf{D}(\underline{\mathbf{M N S T}})$ by the smallest localising subcategory containing all complexes of the form:

(CI) for $\mathfrak{M} \in \underline{\text { MCor }}$,

$$
\mathbb{Z}_{\text {tr }}(\mathfrak{M} \otimes \square) \rightarrow \mathbb{Z}_{\text {tr }}(\mathfrak{M})
$$

Note that complexes of the following form are quasi-isomorphic to zero in $D$ (MNST):

$(\mathrm{MV})$ for $\mathfrak{M} \in \underline{\mathbf{M} C o r}$ and an elementary Nisnevich $\operatorname{cover}^{2}(\mathfrak{U}, \mathfrak{V})$ of $\mathfrak{M}$,

$$
\mathbb{Z}_{\text {tr }}\left(\mathfrak{U} \times_{\mathfrak{M}} \mathfrak{V}\right) \rightarrow \mathbb{Z}_{\text {tr }}(\mathfrak{U}) \oplus \mathbb{Z}_{\text {tr }}(\mathfrak{V}) \rightarrow \mathbb{Z}_{\text {tr }}(\mathfrak{M})
$$

We define $\mathbf{M D M}^{\text {eff }}$ to be the smallest subcategory of $\underline{\mathbf{M D M}} \mathbf{M}^{\mathrm{eff}}$ containing the objects $\underline{\mathbf{M}}\left(\bar{M}, M^{\infty}\right)$ for modulus pair $\left(\bar{M}, M^{\infty}\right)$ such that $\bar{M}$ is proper, and closed under isomorphisms, direct sums, shifts, and cones.

We have a functor

$$
\underline{\omega}: \underline{\text { MCor } \rightarrow \text { Cor }}
$$

with $\underline{\omega}\left(\bar{M}, M^{\infty}\right)=M^{\circ}:=\bar{M} \backslash M^{\infty}$. This functor $\underline{\omega}$ induces a triangulated functor

$$
\underline{\omega}_{\text {eff }}: \underline{\mathbf{M D M}}^{\mathrm{eff}} \rightarrow \mathbf{D M}^{\mathrm{eff}} \text {. }
$$

Definition 2.1. In Situation 1.1, we define the closed Thom space as

$$
\begin{aligned}
& T h\left(N_{Z} M, c l\right):=\text { Cone }\left(\underline{\mathbf{M}}\left(\mathbb{P}\left(N_{\bar{Z}} \bar{M} \oplus \mathcal{O}\right), \pi^{*} Z^{\infty}+\{\infty\}_{\bar{Z}}\right)\right. \\
&\left.\rightarrow \underline{\mathbf{M}}\left(\mathbb{P}\left(N_{\bar{Z}} \bar{M} \oplus \mathcal{O}\right), \pi^{*} Z^{\infty}\right)\right) .
\end{aligned}
$$

in $\underline{\mathbf{M D M}^{\mathrm{eff}}}$, where $\pi: \mathbb{P}\left(N_{\bar{Z}} \bar{M} \oplus \mathcal{O}\right) \rightarrow \bar{Z}$ is the canonical projection.

Notice that the closed Thom space is a lifting of Voevodsky's Thom spaces in the sense that $\underline{\omega}_{\text {eff }}$ sends $T h\left(N_{Z} M, c l\right)$ to $T h\left(N_{Z \circ} M^{\circ}\right)$.

For a smooth variety and a vector bundle $E$ on $X$, Voevodsky defined the Thom space in $\mathbf{D M}^{\text {eff }}$ :

$$
T h_{X}(E)=\operatorname{Cone}\left(\mathbb{P}_{X}(E \oplus \mathcal{O}) \backslash\{\infty\}_{X} \rightarrow \mathbb{P}_{X}(E \oplus \mathcal{O})\right) .
$$

Remark 2.2. Note that $T h\left(N_{Z} M\right)$ is a direct summand of $\underline{\mathbf{M}}\left(\mathbb{P}\left(N_{\bar{Z}} \bar{M} \oplus \mathcal{O}\right), \pi^{*} Z^{\infty}\right)$ since $\underline{\mathbf{M}}\left(\mathbb{P}(\mathcal{O}), \pi^{*} Z^{\infty}\right) \simeq$ $\underline{\mathbf{M}}\left(\bar{Z}, Z^{\infty}\right)$. Cf. [KS19, Lemma 6]. In fact, by the projective bundle formula [KMSY20, Theorem 7.3.2], the closed Thom spaces are just Tate twists: $\operatorname{Th}\left(N_{Z} M, c l\right) \cong \underline{\mathbf{M}}\left(\bar{Z}, Z^{\infty}\right)(1)[2]$.

Remark 2.3. For any proper birational morphism of schemes $f: X \rightarrow Y$ and effective Cartier divisors $Y^{\infty} \subset Y$ and $X^{\infty}=f^{*} Y^{\infty}$ satisfying $Y \backslash Y^{\infty} \simeq X \backslash X^{\infty}$, there is an isomorphism

$$
\underline{\mathbf{M}}\left(Y, Y^{\infty}\right) \simeq \underline{\mathbf{M}}\left(X, X^{\infty}\right)
$$

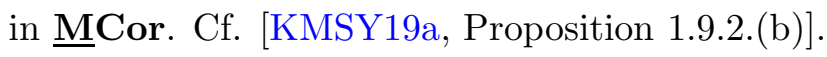

The following basic homological algebra result will be useful.

Lemma 2.4. Consider a commutative diagram.

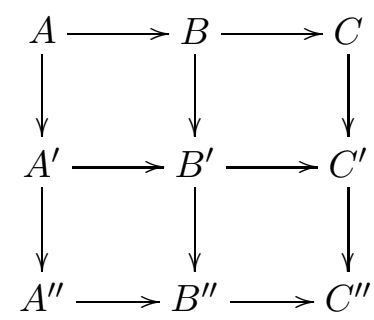

in an additive category $\mathcal{A}$ such that all horizontal and vertical compositions are zero. Suppose we have a triangulated functor $\Phi: K^{b}(\mathcal{A}) \rightarrow T$ to some triangulated category $T$ such that (the complexes associated

\footnotetext{
${ }^{2}$ By elementary Nisnevich cover we mean morphisms $\left\{\left(\bar{U}, U^{\infty}\right) \rightarrow \mathfrak{M},\left(\bar{V}, V^{\infty}\right) \rightarrow \mathfrak{M}\right\}$ such that $\{\bar{U} \rightarrow \bar{M}, \bar{V} \rightarrow \bar{M}\}$ is an elementary Nisnevich cover in Voevodsky's sense, and $U^{\infty}, V^{\infty}$ are the pullbacks of $M^{\infty}$. By $\mathfrak{U} \times \mathfrak{M} \mathfrak{V}$ we mean $\left(\bar{U} \times \bar{M} \bar{V}, p r_{1}^{-1} U^{\infty}+p r_{2}^{-1} V^{\infty}\right)$.
} 
to) all three columns and two of the rows are sent to zero in $T$. Then the (the complex associated to) the third row is sent to zero in $T$ as well.

Proof. Clear.

\section{EXCISION}

In this section we prove some basic excision results and prove that Thom spaces are invariant under change of étale neighbourhood.

Let $M=\left(\bar{M}, M^{\infty}\right)$ and $Z=\left(\bar{Z}, Z^{\infty}\right)$ be as in Situation 1.1. For $n \in \mathbb{Z}_{\geq 0}$ we define a presheaf on $\underline{\text { MCor }}$

$$
C_{n Z}^{M}=\operatorname{Coker}\left(\mathbb{Z}_{\mathrm{tr}}\left(\bar{U}, U^{\infty}\right) \hookrightarrow \mathbb{Z}_{\mathrm{tr}}\left(\bar{M}, M^{\infty}+n \bar{Z}\right)\right)
$$

where $\bar{U}=\bar{M} \backslash \bar{Z}, U^{\infty}=\left.M^{\infty}\right|_{\bar{U}}$ and $\mathbb{Z}_{\mathrm{tr}}\left(\bar{U}, U^{\infty}\right) \rightarrow \mathbb{Z}_{\mathrm{tr}}\left(\bar{M}, M^{\infty}+n \bar{Z}\right)$ is induced by the open immersion $\bar{U} \rightarrow \bar{M}$.

For a morphism $f:\left(\bar{M}, M^{\infty}\right) \rightarrow\left(\bar{N}, N^{\infty}\right)$ induced by a morphism of schemes $\bar{f}: \bar{M} \rightarrow \bar{N}$, we call $f$ minimal if we have $M^{\infty}=\bar{f}^{*} N^{\infty}$.

Proposition 3.1. Let $f:\left(\bar{N}, N^{\infty}\right) \rightarrow\left(\bar{M}, M^{\infty}\right)$ be an étale morphism (i.e., $f$ is induced by an étale morphism $\bar{f}: \bar{N} \rightarrow \bar{M}$ and is minimal). If $\bar{f}^{-1} \bar{Z} \rightarrow \bar{Z}$ is an isomorphism, then for any $n \in \mathbb{Z}_{\geq 0}$, the natural morphism $C_{n f^{-1} Z}^{N} \rightarrow C_{n Z}^{M}$ is a isomorphism in $\underline{\mathbf{M D M}}^{\mathrm{eff}}$.

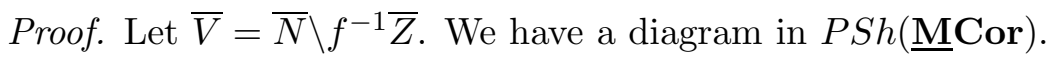

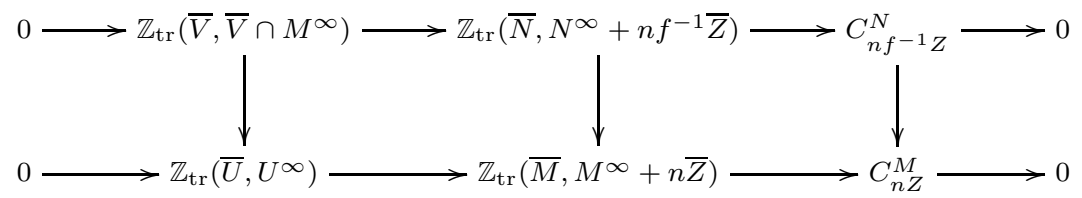

The left hand-side square is homotopy Cartesian in $\underline{\mathbf{M D M}} \mathbf{M}^{\mathrm{eff}}$ by the definition of $\underline{\mathbf{M}} \mathbf{M}^{\mathrm{eff}}$. So we get the claim.

Theorem 3.2. Let $f:\left(\bar{N}, N^{\infty}\right) \rightarrow\left(\bar{M}, M^{\infty}\right)$ be an étale morphism. If $\bar{f}^{-1} \bar{Z} \rightarrow \bar{Z}$ is an isomorphism,

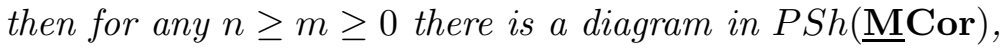

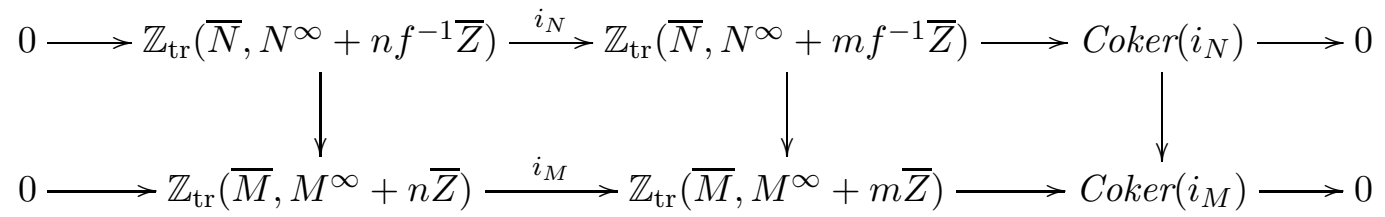

such that Coker $\left(i_{N}\right) \rightarrow \operatorname{Coker}\left(i_{M}\right)$ is an isomorphism in $\mathbf{M D M}^{\mathrm{eff}}$.

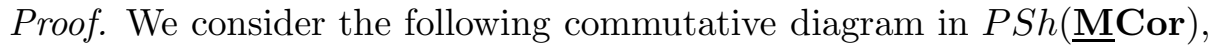

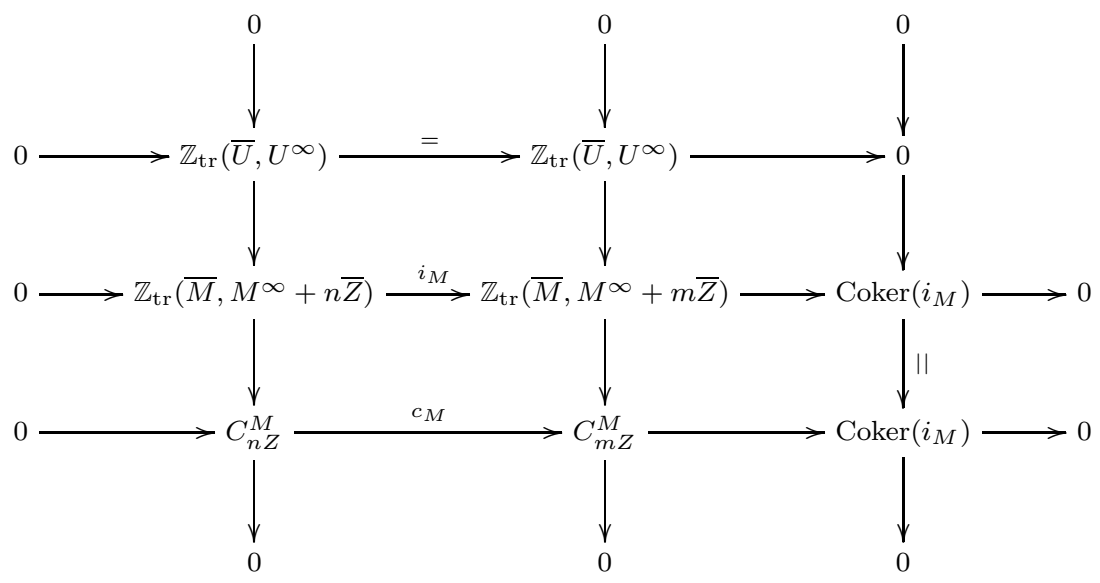


where $i_{M}$ is the natural map and $c_{M}$ is the unique map determined by $i_{M}$. Now all columns and the two top rows are exact. Now by the nine lemma, we get that the bottom row is also exact. The morphisms $i_{M}$ and $\bar{f}$ induce the commutative diagram:

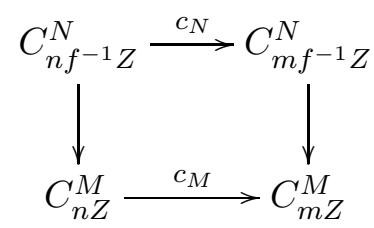

By Proposition 3.1, the vertical morphisms become isomorphisms in $\mathbf{M D M}^{\text {eff }}$. Hence the map between the cokernels of the two horizontal presheaf monomorphims become isomorphisms in $\mathbf{M D M}^{\text {eff }}$.

Corollary 3.3. In the situation of Theorem 3.2 Thom spaces are isomorphic

$$
T h\left(N_{f^{-1} \bar{Z}} N, c l\right) \simeq T h\left(N_{\bar{Z}} M, c l\right)
$$

Proof. In the situation of Theorem 3.2 for any $n \in\{0,1\}$ the natural morphism

$$
\bar{f}:\left(\mathbb{P}\left(N_{f^{-1}} \bar{Z} \bar{N} \oplus \mathcal{O}\right), \pi^{\prime *} Z^{\prime \infty}+n\{\infty\}_{f^{-1}} \bar{Z}\right) \rightarrow\left(\mathbb{P}\left(N_{\bar{Z}} \bar{M} \oplus \mathcal{O}\right), \pi^{*} Z^{\infty}+n\{\infty\}_{\bar{Z}}\right)
$$

is minimal étale morphism where $Z^{\prime \infty}:=f^{-1} \bar{Z} . N^{\infty}$ and $\pi^{\prime}$ is the projection $\mathbb{P}\left(N_{f^{-1}} \overline{\bar{Z}} \oplus \mathcal{O}\right) \rightarrow f^{-1} \bar{Z}$. Moreover $\bar{f}$ induces an isomorphism $\{\infty\}_{f^{-1} \bar{Z}} \simeq\{\infty\}_{\bar{Z}}$ since $f^{-1} \bar{Z} \simeq \bar{Z}$. By Proposition 3.1 and Theorem 3.2 we obtain the claim.

\section{Blow up FORMula With Weight}

4.1. Introduction. Kelly-Saito proved a blow up formula for motives with modulus (see [KS19]), but to construct tame Gysin map we need another formula, namely, Theorem 4.2. In this section, we calculate some motives of Fano surfaces with modulus, after that we have constructed the formula which we need. We begin with the notation that we will need to perform the deformation to the normal cone technique.

Notation 4.1. In the Situation 1.1, we use the following notations.

$$
\begin{aligned}
& M:=\left(\bar{M}, M^{\infty}\right), \\
& Z:=\left(\bar{Z}, Z^{\infty}\right), \\
& \bar{B}_{M}^{(\bar{Z})} \stackrel{\pi_{M}}{\longrightarrow} \times \mathbb{P}^{1}: \text { the blow up of } \bar{M} \times \mathbb{P}^{1} \text { at } \bar{Z} \times\{0\} \\
& B_{M}^{\infty}:=\pi_{M}^{*}\left(M^{\infty} \times \mathbb{P}^{1}+\bar{M} \times\{\infty\}\right), \\
& W_{M}: \text { the strict transform of } \bar{Z} \times \mathbb{P}^{1} \text { w.r.t. } \bar{B}_{M} \rightarrow \bar{M} \times \mathbb{P}^{1}, \\
& B_{M, c l}^{(\bar{Z})}:=\left(\bar{B}_{M}^{(\bar{Z})}, B_{M}^{\infty}+W_{M}\right), \\
& \bar{U}_{M}:=\bar{M} \times \mathbb{P}^{1} \backslash \bar{Z} \times \mathbb{P}^{1}, \\
& \bar{E}_{M}^{(\bar{Z})}:=\text { the exceptional divisor of } \pi_{M}, \\
& E_{M, c l}^{(\bar{Z})}:=\left(\bar{E}_{M},\left(\bar{E}_{M} \cap B_{M}^{\infty}\right)+\left(\bar{E}_{M} \cap W_{M}\right)\right) .
\end{aligned}
$$

The goal of this section is to prove the following theorem.

Theorem 4.2. In the notation of Situation 1.1, there exist a distinguished triangle in $\underline{\mathbf{M D M}} \mathbf{e f f}^{\mathrm{e}}$.

$$
\underline{\mathbf{M}}\left(E_{M, c l}^{(\bar{Z})}\right) \rightarrow \underline{\mathbf{M}}(Z) \oplus \underline{\mathbf{M}}\left(B_{M, c l}^{(\bar{Z})}\right) \rightarrow \underline{\mathbf{M}}(M \otimes \bar{\square}) \stackrel{+}{\rightarrow} .
$$


4.2. Special case. Let $H_{0}, H_{1}, H_{2}$ be the hyperplanes on $\mathbb{P}^{2}$ given by $\{[0: \star: \star]\},\{[\star: 0: \star]\},\{[\star: \star: 0]\}$. We set $b: B \rightarrow \mathbb{P}^{2}$ to be the blow up of $\mathbb{P}^{2}$ along $H_{0} \cap H_{1}$, and set $\widetilde{H_{0}}, \widetilde{H_{1}}, \widetilde{H_{2}}$ to be the strict transforms of $H_{0}, H_{1}, H_{2}$. We set

$$
B_{c l}:=\left(B, \widetilde{H_{0}}+\widetilde{H_{2}}\right) \quad \text { and } \quad E_{c l}:=\left(E, E \cap \widetilde{H_{0}}\right),
$$

where $E$ is the exceptional divisor of the blow up.

Proposition 4.3. There is a split distinguish triangle

$$
\underline{\mathbf{M}}\left(E_{c l}\right) \stackrel{\left[\begin{array}{ll}
p & i
\end{array}\right]}{\longrightarrow} \underline{\mathbf{M}}(\operatorname{Spec} k) \oplus \underline{\mathbf{M}}\left(B_{c l}\right) \stackrel{\left[\begin{array}{c}
j \\
-b
\end{array}\right]}{\longrightarrow} \underline{\mathbf{M}}\left(\mathbb{P}^{2}, H_{2}\right) \underset{0}{\stackrel{+}{\longrightarrow}}
$$

in $\underline{\mathbf{M D M}}^{\mathrm{eff}}$, where $i, j$ are the natural closed immersions and $p$ is the natural projection $E \rightarrow$ Spec $k$.

Proof. Since $B_{c l}$ has a projection to $E_{c l}$ which is a cube bundle, $i$ is an isomorphism. Additionally $j$ is also an isomorphism since $\left(\mathbb{P}^{2}, H_{2}\right)$ is contractible [KS19, Lemma 10].

\subsection{Proof of Theorem 4.2.}

Theorem 4.4. There is a distinguish triangle.

$$
\underline{\mathbf{M}}\left(E_{\left(\mathbb{A}^{1}, \emptyset\right), c l}^{(\{0\})}\right) \rightarrow \underline{\mathbf{M}}(\{0\}) \oplus \underline{\mathbf{M}}\left(B_{\left(\mathbb{A}^{1}, \emptyset\right), c l}^{(\{0\})}\right) \rightarrow \underline{\mathbf{M}}\left(\left(\mathbb{A}^{1}, \emptyset\right) \otimes \bar{\square}\right) \stackrel{+}{\rightarrow} .
$$

A $\log$ version of the argument below appeared independently in Binda-Park-Østvær (see [BPØ20, Proposition 7.2.5]).

Proof. We set $T$ to be the blow up of $\mathbb{P}^{2}$ at $H_{0} \cap H_{2}$, let $f$ be the exceptional divisor, and $h_{i}$ be the strict transforms of the $H_{i}$. We set $T^{\prime}$ to be the blow up of $T$ at $h_{0} \cap h_{1}$, let $e$ be the exceptional divisor, and the $\widetilde{h_{i}}$ be strict transforms of the $h_{i}$ and $\widetilde{f}$ be the strict transform of $f$. In particular, $T^{\prime}$ is same as the blow up of $B$ at $\widetilde{H_{0}} \cap \widetilde{H}_{2}$. The fans of these toric varieties are as follows:

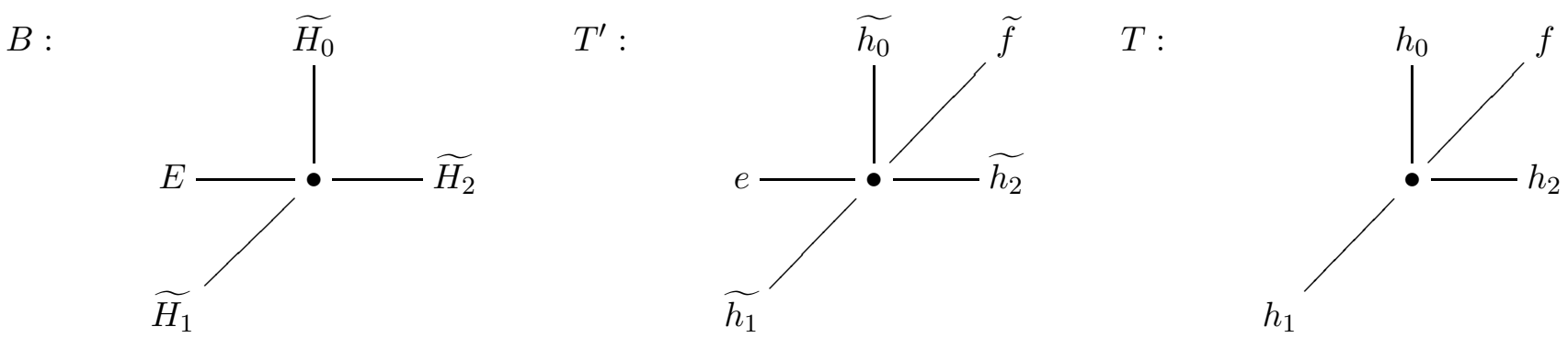

The following triangle is isomorphic to it in Proposition 4.3, since this is obtained by blowing up inside the modulus.

$$
\underline{\mathbf{M}}\left(E_{c l}\right) \rightarrow \underline{\mathbf{M}}(\operatorname{Spec} k) \oplus \underline{\mathbf{M}}\left(T^{\prime}, \widetilde{h_{2}}+\widetilde{h_{0}}+\widetilde{f}\right) \rightarrow \underline{\mathbf{M}}\left(T, h_{2}+f\right) \underset{0}{\stackrel{+}{\rightarrow}}
$$

Notice that there is a canonical isomorphism of toric surfaces $T \backslash h_{2} \cong \mathbf{A}^{1} \times \mathbf{P}^{1}$ inducing an isomorphism of modulus pairs $\left(T \backslash h_{2}, f \backslash\left(f \cap h_{2}\right)\right) \cong\left(\mathbf{A}^{1}, \emptyset\right) \otimes \bar{\square}$. Furthermore, pulling back the square that give rise to (4.2) along $\mathbf{A}^{1} \times \mathbf{P}^{1} \rightarrow T$ produces the triangle in the statement.

Since $\left\{T \backslash h_{2} \rightarrow T, T \backslash h_{0} \rightarrow T\right\}$ is a Zariski covering, by Mayer-Vietoris, to show that the triangle in the statement is distinguished, it suffices to show that the triangle associated to $T, T \backslash h_{0}$, and $T \backslash\left(h_{0} \cup h_{2}\right)$ is distinguished, cf. Lem. 2.4. We have just seen that the triangle associated to $T$ is isomorphic distinguish triangle (4.2). On the other hand, since the centre of the blowup is contained in $h_{0}$, the triangle coming from $T \backslash h_{0}$ and $T \backslash\left(h_{0} \cup h_{2}\right)$ is trivially distinguished.

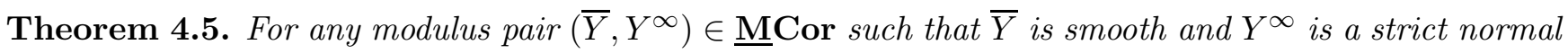
crossings divisor, there is a distinguish triangle:

$$
\underline{\mathbf{M}}\left(E_{Y \otimes\left(\mathbb{A}^{1}, \emptyset\right), *}^{(Y \otimes\{0)}\right) \rightarrow \underline{\mathbf{M}}(Y \otimes\{0\}) \oplus \underline{\mathbf{M}}\left(B_{Y \otimes\left(\mathbb{A}^{1}, \emptyset\right), *}^{(Y \otimes\{0)}\right) \rightarrow \underline{\mathbf{M}}\left(Y \otimes\left(\mathbb{A}^{1}, \emptyset\right) \otimes \bar{\square}\right) \stackrel{+}{\rightarrow} .
$$


Proof. Since

$$
B_{Y \otimes\left(\mathbb{A}^{1}, \emptyset\right), c l}^{(Y \otimes\{0)}=Y \otimes B_{\left(\mathbb{A}^{1}, \emptyset\right), c l}^{(\{0\})},
$$

the triangle in the statement is the triangle from Theorem 4.4 tensored by $\left(\bar{Y}, Y^{\infty}\right)$.

Situation 4.6. Let $f:\left(\bar{N}, N^{\infty}\right) \rightarrow\left(\bar{M}, M^{\infty}\right)$ be an étale morphism (i.e., $f$ is induced by an étale morphism $\bar{f}: \bar{N} \rightarrow \bar{M}$ and is minimal) such that $f$ induces an isomorphism $f^{-1} \bar{Z} \rightarrow \bar{Z}$.

Notation 4.7. In Situation 4.6, we pullback everything from Notation 4.1 along $f$. That is, we set

$$
N, f^{-1} Z, \bar{B}_{N}^{\left(f^{-1} \bar{Z}\right)}, \pi_{N}, B_{N}^{\infty}, W_{N}, B_{N, c l}^{\left(f^{-1} \bar{Z}\right)}, \bar{U}_{N}, U_{N}, \bar{E}_{N}^{\left(f^{-1} \bar{Z}\right)}, E_{N, c l}^{\left(f^{-1} \bar{Z}\right)}
$$

to be the pullbacks of

$$
M, Z, \bar{B}_{M}^{(\bar{Z})}, \pi_{M}, B_{M}^{\infty}, W_{M}, B_{M, o p}^{(\bar{Z})}, B_{M, c l}^{(\bar{Z})}, \bar{U}_{M}, U_{M}, \bar{E}_{M}^{(\bar{Z})}, E_{M, o p}^{(\bar{Z})}, E_{M, c l}^{(\bar{Z})}
$$

along $\bar{f}: \bar{N} \rightarrow \bar{M}$. Explicitly,

$$
\begin{aligned}
& N:=\left(\bar{N}, N^{\infty}\right), \\
& f^{-1} Z:=\left(f^{-1} \bar{Z}, f^{-1} Z \cdot \bar{N} N^{\infty}\right)
\end{aligned}
$$

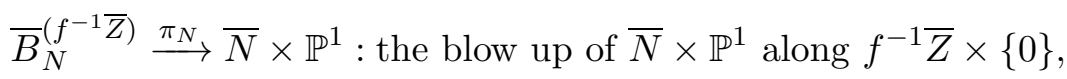

$$
\begin{aligned}
& B_{N}^{\infty}:=\pi_{N}^{*}\left(N^{\infty} \times \mathbb{P}^{1}+\bar{N} \times\{\infty\}\right) \\
& W_{N} \text { : the strict transform of } f^{-1} \bar{Z} \times \mathbb{P}^{1} \text { w.r.t. } \bar{B}_{N} \rightarrow \bar{N} \times \mathbb{P}^{1} \text {, } \\
& B_{N, c l}^{\left(f^{-1} \bar{Z}\right)}:=\left(\bar{B}_{N}, B_{N}^{\infty}+W_{N}\right), \\
& \bar{U}_{N}:=\bar{N} \times \mathbb{P}^{1} \backslash f^{-1} \bar{Z} \times \mathbb{P}^{1}, \\
& U_{N}:=\left(\bar{U}_{N}, \bar{U}_{N} \cap\left(N^{\infty} \times \mathbb{P}^{1}+\bar{N} \times\{\infty\}\right)\right) \\
& \bar{E}_{N}^{\left(f^{-1} \bar{Z}\right)} \text { : the exceptional divisor of } \pi_{N} \text {, } \\
& E_{N, c l}^{\left(f^{-1} \bar{Z}\right)}:=\left(\bar{E}_{N}, \bar{E}_{N} \cap B_{N}^{\infty}+\left(W_{N} \cap \bar{E}_{N}\right)\right) .
\end{aligned}
$$

Proposition 4.8. In Situation 4.6 and Notation 4.7 ,

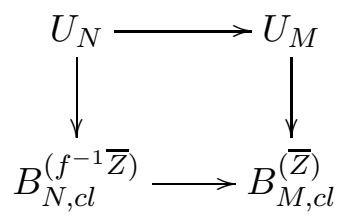

is elementary Nisnevich square.

Proof. All morphisms in the square are minimal. By definitions, $\left(B_{M, c l}^{(\bar{Z})} \backslash U_{M}\right)=\left(W_{M} \cup \bar{E}_{M}\right),\left(B_{N, c l}^{\left(f^{-1} \bar{Z}\right)} \backslash U_{N}\right)=$ $\left(W_{N} \cup \bar{E}_{N}\right)$.

Corollary 4.9. In Situation 4.6 and Notation 4.7, the image under the functor $\underline{\mathbf{M}}$ of the square

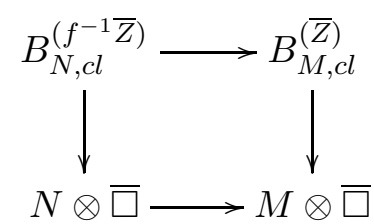

is a homotopy Cartesian in $\underline{\mathbf{M D M}} \mathbf{M}^{\mathrm{eff}}$.

Notation 4.10. In Notation 4.1 Consider the following "weighted" blowup formulas.

$(W B U)_{Z \rightarrow M}^{c l}$ : the object $\underline{\mathbf{M}}\left(E_{M, c l}^{(\bar{Z})} \rightarrow Z \oplus B_{M, c l}^{(\bar{Z})} \rightarrow M \otimes \bar{\square}\right)$ is isomorphic to zero in $\underline{\mathbf{M D M}} \mathbf{M}^{\mathrm{eff}}$. 
Proposition 4.11. In Situation 4.6, $(W B U)_{Z \rightarrow M}^{*}$ is true if and only if $(W B U)_{f^{-1} Z \rightarrow N}^{*}$ is true.

Proof. The following diagram commutes in $\underline{\mathbf{M C o r}}$.

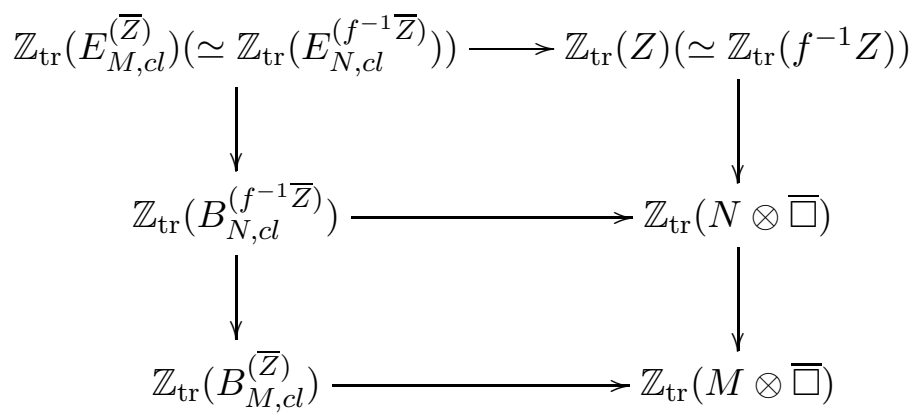

By Corollary 4.9 we know the lower square is a homotopy Cartesian in $\underline{\text { MDM }}{ }^{\text {eff }}$, so the outer square is a homotopy Cartesian iff the upper square is.

The following lemma is proved in [KS19].

Lemma 4.12 ([KS19, Lemma 8]). In Situation 1.1, up to replacing $\bar{M}, \bar{Z}, M^{\infty}$ by $\bar{V}, \bar{V} \cap \bar{Z}, \bar{V} \cap M^{\infty}$ for some open neighborhood $x \in \bar{V}$, there exists an étale morphism $q: \bar{M} \rightarrow \mathbb{A}^{m}$ such that $\bar{Z}=q^{-1}\left(\mathbb{A}^{m-1} \times\right.$ $\{0\})$ and $M^{\infty}=q^{-1}\left(\left\{T_{1}^{d_{1}} \ldots T_{s}^{d_{s}}=0\right\}\right)$ where $T_{i}$ are the coordinates of $\mathbb{A}^{m}$.

Now we have enough pieces to prove Theorem 4.2.

Proof of Theorem 4.2. For any open covering $\left\{\bar{U}_{i} \rightarrow \bar{M}\right\}_{i}$, by the Mayer-Vietoris sequence we obtain that $(W B U)_{Z \rightarrow M}^{*}$ is true if $(W B U)_{Z \cap \bar{U} \rightarrow\left(\bar{U}, \bar{U} \cap M^{\infty}\right)}^{*}$ is true for all open sub schemes $\bar{U} \subset \bar{U}_{i}$ for all $i$. By Proposition 4.11 and Lemma 4.12 we can reduce the claim to the case $(W B U)_{Z \otimes\{0\} \rightarrow Z \otimes\left(\mathbb{A}^{1}, \emptyset\right)}^{*}$, but this was proved in Theorem 4.5.

\section{Construction of the Tame Gysin map}

5.1. Notation. We continue with $\mathfrak{M}=\left(\bar{M}, M^{\infty}\right)$ and $\mathfrak{Z}=\left(\bar{Z}, Z^{\infty}\right)$ satisfying the hypotheses of Situation 1.1. We furthermore drop all the indexes " $M$ " from the notation of Notation 4.1. So

$$
\bar{B}: \text { is the blow-up of } \bar{M} \times \mathbb{P}^{1} \text { along } \bar{Z} \times\{0\} \text {, and }
$$

$$
E: \text { is the exceptional divisor of } q \text {, }
$$

so we have a Cartesian square

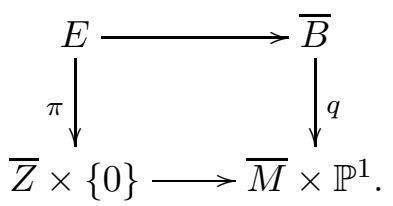

We put

$$
\begin{gathered}
\mathfrak{B}:=\left(\bar{B}, q^{*}\left(M^{\infty} \times \mathbb{P}^{1}+\bar{M} \times\{\infty\}\right)\right), \\
\mathfrak{B}_{Z, c l}:=\left(\bar{B}, q^{*}\left(M^{\infty} \times \mathbb{P}^{1}+\bar{M} \times\{\infty\}\right)+\left(\overline{\bar{Z} \times \mathbb{P}^{1}}\right)\right) \\
\mathfrak{E}_{Z, c l}:=\left(\mathbb{P}\left(N_{Z} M \oplus \mathcal{O}\right), \pi^{*} Z^{\infty}+\mathbb{P}(0 \oplus \mathcal{O})\right) .
\end{gathered}
$$

Theorem 5.1. There is a distinguished triangle in $\underline{\mathbf{M D M}} \mathbf{M}^{\mathrm{eff}}$

$$
\underline{\mathbf{M}}\left(\mathfrak{E}_{Z, c l}\right) \rightarrow \underline{\mathbf{M}}\left(B_{Z, c l}\right) \oplus \underline{\mathbf{M}}(\mathfrak{Z}) \rightarrow \underline{\mathbf{M}}(\mathfrak{M} \otimes \bar{\square}) \stackrel{+}{\rightarrow}
$$

and isomorphism

$$
T h\left(N_{Z} M, c l\right)=\operatorname{Cone}\left(\underline{\mathbf{M}}\left(\mathfrak{E}_{Z, c l}\right) \rightarrow \underline{\mathbf{M}}(\mathfrak{E})\right) \simeq \operatorname{Cone}\left(\underline{\mathbf{M}}\left(\mathfrak{B}_{Z, c l}\right) \rightarrow \underline{\mathbf{M}}(\mathfrak{B})\right) .
$$


Proof. The first claim is Theorem 4.2, the second claim follows from the first and the blow up formula

$$
\underline{\mathbf{M}}(\mathfrak{E}) \rightarrow \underline{\mathbf{M}}(\mathfrak{B}) \oplus \underline{\mathbf{M}}(\mathfrak{Z}) \rightarrow \underline{\mathbf{M}}(\mathfrak{M} \otimes \bar{\square}) \stackrel{+}{\rightarrow}
$$

proved in [KS19, Theorem, page 1].

5.2. Geometrical study. We write $i_{1}$ for the natural embedding of schemes $\bar{M} \times\{1\}$ to $\bar{B}$. The embedding $i_{1}$ defines $i: \mathfrak{M} \rightarrow \mathfrak{B}$ and $\tilde{i}: \mathfrak{M}_{Z, c l} \rightarrow \mathfrak{B}_{Z, c l}$ in $\underline{\text { MCor. }}$

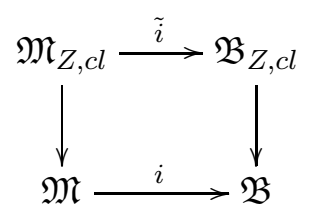

The diagram gives us the morphism $\mathbb{Z}_{\mathrm{tr}}\left(\mathfrak{M} / \mathfrak{M}_{Z, c l}\right) \rightarrow \mathbb{Z}_{\mathrm{tr}}\left(\mathfrak{B} / \mathfrak{B}_{Z, c l}\right)$ in $P S h(\underline{\mathbf{M C o r}})$ where we write

$$
\mathbb{Z}_{\mathrm{tr}}\left(\mathfrak{M} / \mathfrak{M}_{Z, c l}\right):=\operatorname{coker}\left(\mathbb{Z}_{\mathrm{tr}}\left(\mathfrak{M}_{Z, c l}\right) \rightarrow \mathbb{Z}_{\mathrm{tr}}(\mathfrak{M})\right)
$$

etc., in $P S h(\underline{\text { MCor }})$. Note that since $\mathfrak{M}_{Z, c l} \rightarrow \mathfrak{M}$ are monomorphisms, the image of $\mathbb{Z}_{\text {tr }}\left(\mathfrak{M} / \mathfrak{M}_{Z, c l}\right)$ in $\underline{M D M}^{\text {eff }}$ is the cone of the image of $\mathbb{Z}_{\mathrm{tr}}\left(\mathfrak{M}_{Z, c l}\right) \rightarrow \mathbb{Z}_{\mathrm{tr}}(\mathfrak{M})$. Composing with the isomorphism

$$
\mathbb{Z}_{\operatorname{tr}}\left(\mathfrak{B} / \mathfrak{B}_{Z, c l}\right) \stackrel{\leftarrow}{\leftarrow} \operatorname{tr}\left(\mathfrak{E} / \mathfrak{E}_{Z, c l}\right)=T h\left(N_{Z} M, c l\right)
$$

from Theorem 5.1, one gets a morphism:

$$
\beta(\mathfrak{M} / Z, c l): \underline{\mathbf{M}}\left(\mathfrak{M} / \mathfrak{M}_{Z, c l}\right) \rightarrow \mathbb{Z}_{\mathrm{tr}}\left(\mathfrak{B} / \mathfrak{B}_{Z, c l}\right) \rightarrow T h\left(N_{Z} M, c l\right) .
$$

We call this morphism the closed Gysin map associated with $\mathfrak{M}$ and $Z$.

Lemma 5.2. We have the following.

(0) $\beta\left(\left(\mathbb{A}^{1}, \emptyset\right) /\{0\}, c l\right): \underline{\mathbf{M}}\left(\left(\mathbb{A}^{1}, \emptyset\right) /\left(\mathbb{A}^{1},\{0\}\right)\right) \rightarrow T h\left(N_{\{0\}}\left(\mathbb{A}^{1}, \emptyset\right), c l\right)$ is an isomorphism.

(1) For any étale morphism e $: \mathfrak{M}^{\prime}=\left(\bar{M}^{\prime}, e^{*} M^{\infty}\right) \rightarrow \mathfrak{M}$, set $\mathfrak{Z}^{\prime}=\left(e^{-1} \bar{Z}, e^{*} Z^{\infty}\right)$. Then the diagram

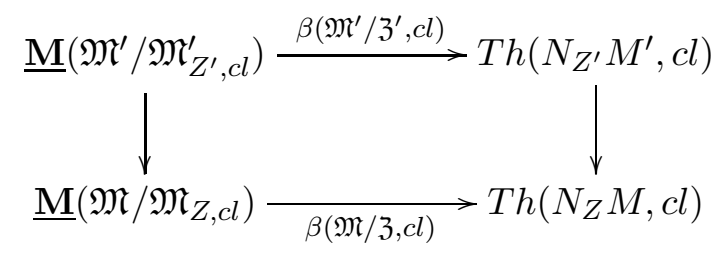

commutes.

(2) For any modulus pair $\mathfrak{Y}=\left(\bar{Y}, Y^{\infty}\right)$ with $\bar{Y}$ smooth and $Y^{\infty}$ a strict normal crossings divisor, we have

$$
\beta(\mathfrak{M} \otimes \mathfrak{Y} / \mathfrak{Z} \otimes \mathfrak{Y}, c l)=\beta(\mathfrak{M} / \mathfrak{Z}, c l) \otimes I d_{\underline{M}(\mathfrak{Y})}
$$

Proof. Part 1. We take

$$
\bar{B}^{\prime} \text { : blow-up of } \bar{M}^{\prime} \times \mathbb{P}^{1} \text { with along } e^{-1} \bar{Z} \times\{0\},
$$

and

$$
\begin{gathered}
\mathfrak{B}^{\prime}=\left(\bar{B}^{\prime}, q^{\prime *}\left(e^{*} M^{\infty} \times \mathbb{P}^{1}\right)+q^{\prime *}\left(\bar{M}^{\prime} \times\{\infty\}\right)\right), \\
\mathfrak{B}_{\mathfrak{Z}^{\prime}, c l}^{\prime}=\left(\bar{B}^{\prime}, q^{\prime *}\left(e^{*} M^{\infty} \times \mathbb{P}^{1}\right)+q^{\prime *}\left(\bar{M}^{\prime} \times\{\infty\}\right)+\left(\widetilde{\bar{Z}^{\prime} \times \mathbb{P}^{1}}\right)\right)
\end{gathered}
$$


Since the morphism $e$ is étale, $e^{-1} \bar{Z}$ is also smooth. Now there is a natural map $\mathfrak{B}^{\prime} \rightarrow \mathfrak{B}$, and we have the following commutative diagram in $\underline{\mathbf{M C}}$ Cor.

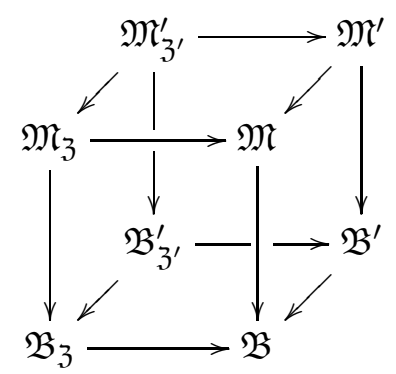

The diagram gives us the commutative diagram in PSh( $\underline{\mathbf{M C}} \mathbf{C o r})$.

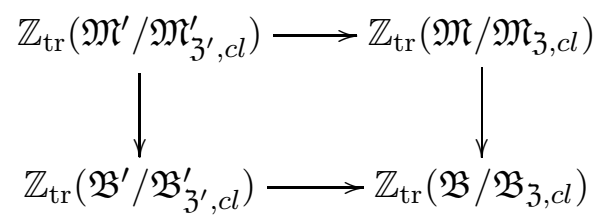

The same argument shows that the square

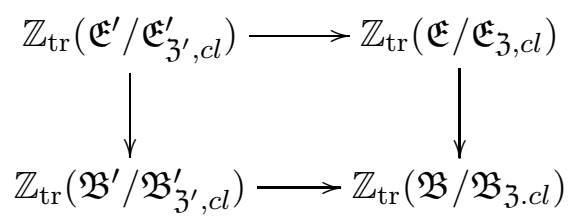

is commutative.

Part 2. The blow-up of $\bar{M} \times \bar{Y} \times \mathbb{P}^{1}$ along $\bar{Z} \times \bar{Y} \times\{0\}$ is isomorphic to $\bar{B} \times \bar{Y}$, so the proof is completed.

Part 0 . Set $\eta_{o p}$ resp. $\eta_{c l}$ to be the composition of the 1 -section $\mathbb{A}^{1} \times\{1\} \hookrightarrow \bar{B}_{\{0\}}$ resp. $\left(\mathbb{A}^{1} \backslash\{0\}\right) \times\{1\} \hookrightarrow$ $\bar{B}_{\{0\}}$, and the retraction $\bar{B}_{\{0\}} \rightarrow \bar{E}_{\{0\}}$ (see the diagrams below on the left). Note that these are open immersions. Let $\eta_{0, c l}, \eta_{1}$ be the induced morphisms on modulus pairs (see the square below on the right).

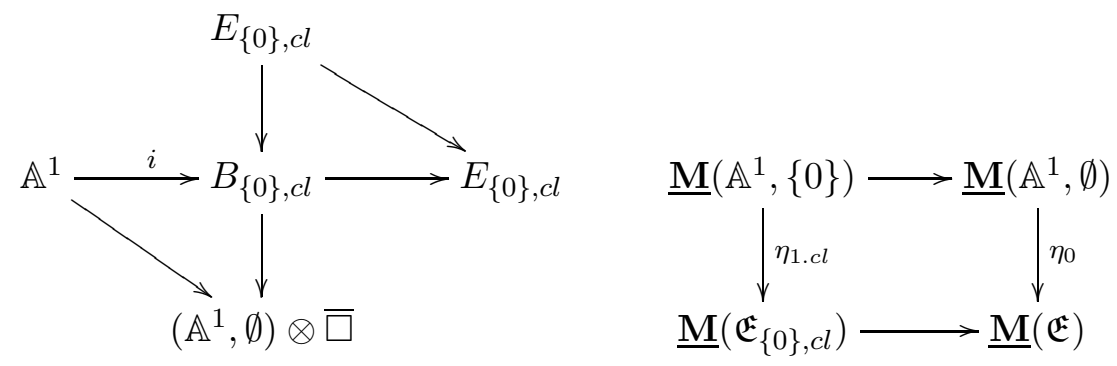

Since both of right side squares satisfy the condition of Proposition 3.1, these squares are homotopy Cartesian in $\underline{\mathbf{M D M}^{\mathrm{eff}}}$.

\section{Proof OF MAIN THEOREMS}

In this section, we use the notation of Section 5.1, and we prove that the Gysin maps defined in Section 5.2

$$
\beta(\mathfrak{M} / \mathfrak{Z}, c l): \underline{\mathbf{M}}\left(\mathfrak{M} / \mathfrak{M}_{\mathfrak{Z}, c l}\right) \rightarrow T h\left(N_{Z} M, c l\right)
$$

are isomorphisms.

Lemma 6.1. The Gysin maps $\beta(\mathfrak{M} / \mathfrak{Z}, *)$ is an isomorphism if there is an open Zariski cover $\left\{\bar{V}_{i} \rightarrow\right.$ $\bar{M}\}_{i=1}^{l}$ such that for all $i$, the Gysin maps $\beta\left(\left(\bar{V}, \bar{V} \cap M^{\infty}\right) /\left(\bar{V} \cap \bar{Z}, \bar{V} \cap M^{\infty} \cap \bar{Z}\right)\right)$ associated to the intersections $\bar{V}=\cap_{j \in J} \bar{V}_{j}$ are isomorphisms for all nonempty $J \subseteq I$. 
Proof. By induction on $l$ it suffices to consider the $l=2$ case. We take an open covering $\bar{V}_{1} \cup \bar{V}_{2}=\bar{M}_{\text {. }}$ Now we set

$$
\begin{gathered}
\mathfrak{V}_{i}=\left(\bar{V}_{i}, \bar{V}_{i} \cap M^{\infty}\right), \quad \mathfrak{V}_{\mathfrak{Z}, i, c l}=\left(\bar{V}_{i}, \bar{V}_{i} \cap M^{\infty}+\bar{V}_{i} \cap \bar{Z}\right), \\
\bar{V}_{12}=\bar{V}_{1} \cap \bar{V}_{2}, \quad \mathfrak{V}_{12}=\left(\bar{V}_{12}, \bar{V}_{12} \cap M^{\infty}\right), \\
\mathfrak{V}_{\mathfrak{Z}, 12, c l}=\left(\bar{V}_{12}, \bar{V}_{12} \cap M^{\infty}+\bar{V}_{12} \cap \bar{Z}\right) .
\end{gathered}
$$

We have the following diagram in $\operatorname{PSh}(\underline{\mathbf{M C}} \mathbf{C o r})$,

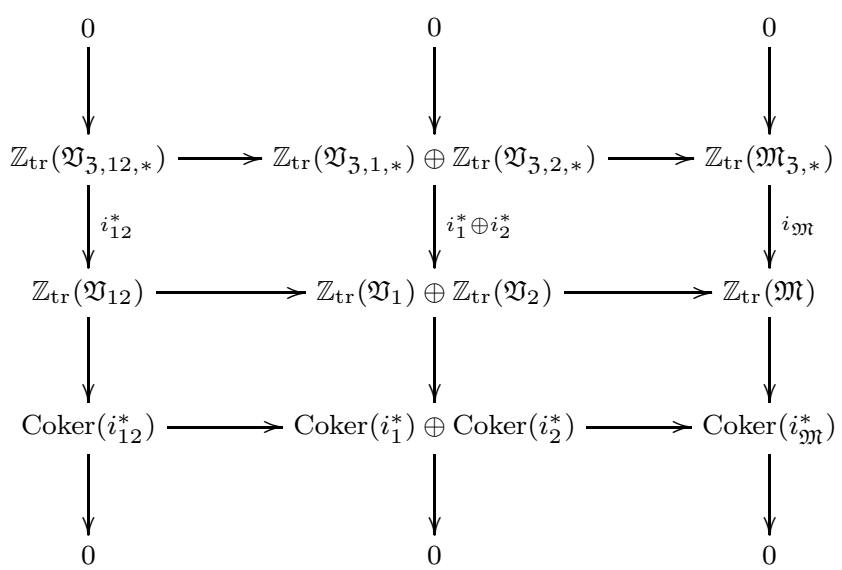

where the compositions of all columns and the two top rows are zero, and the bottom row maps are uniquely determined by the middle row maps.

By Lemma 2.4 we get the following distinguish triangle in $\underline{\mathbf{M D M}} \mathbf{M}^{\mathrm{eff}}$

$$
\underline{\mathbf{M}}\left(\mathfrak{V}_{\mathfrak{Z}, 12, *} / \mathfrak{V}_{\mathfrak{Z}, 12, *}\right) \rightarrow \underline{\mathbf{M}}\left(\mathfrak{V}_{\mathfrak{Z}, 1, *} / \mathfrak{V}_{\mathfrak{Z}, 1, *}\right) \oplus \underline{\mathbf{M}}\left(\mathfrak{V}_{\mathfrak{Z}, 2, *} / \mathfrak{V}_{\mathfrak{Z}, 2, *}\right) \rightarrow \underline{\mathbf{M}}\left(\mathfrak{M}_{\mathfrak{Z}} / \mathfrak{M}_{\mathfrak{Z}}\right) \stackrel{+}{\rightarrow} .
$$

Same argument can be applied for Thom space, so we obtain the following distinguish triangle in $\mathrm{MDM}^{\text {eff }}$

$$
T h\left(N_{Z_{12}} \mathfrak{V}_{12}, *\right) \rightarrow T h\left(N_{Z_{1}} \mathfrak{V}_{1}, *\right) \oplus T h\left(N_{Z_{2}} \mathfrak{V}_{2}, *\right) \rightarrow T h\left(N_{Z} \mathfrak{M}, *\right) \stackrel{+}{\rightarrow} .
$$

By Lemma 5.2, we know that Gysin maps are compatible with open immersions, so the proof follows from the triangulated category axioms.

Lemma 6.2. In the situation Theorem 3.2, $\beta\left(\left(\bar{N}, N^{\infty}\right) /\left(f^{-1} \bar{Z}, Z^{\prime \infty}\right), c l\right)$ is an isomorphism if and only if $\beta\left(\left(\bar{M}, M^{\infty}\right) /\left(\bar{Z}, Z^{\infty}\right), c l\right)$ is isomorphism.

Proof. by Lemma 3.2. (1), we have the following commutative diagram

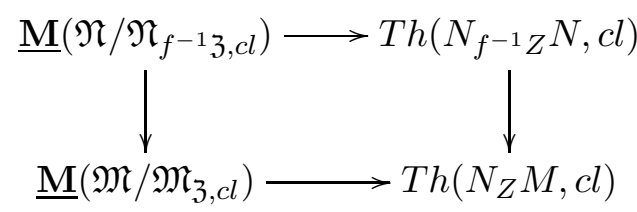

where the vertical maps are isomorphisms by Theorem 3.2 and Corollary 3.3. So if one of the horizon maps is an isomorphism then the other is also an isomorphism.

Now we have a proof of the main theorem.

Proof that the Gysin triangles are distinguished in $\underline{\mathbf{M D M}}^{\mathrm{eff}}$. It suffices to show the Gysin morphisms are isomorphisms. By Lemma 6.1, Lemma 6.2, and Lemma 4.12, we can assume that there is an étale map $\bar{f}: \bar{M} \rightarrow \mathbb{A}^{m}$ such that $M^{\infty}=\bar{f}^{*} E$ and $\bar{Z}=\bar{f}^{*}\left(\mathbb{A}^{m-1} \times\{0\}\right)$ where

$$
\left(\mathbb{A}^{1}, d_{1}\{0\}\right) \otimes\left(\mathbb{A}^{1}, d_{2}\{0\}\right) \otimes \cdots \otimes\left(\mathbb{A}^{1}, \emptyset\right)=\left(\mathbb{A}^{m}, E\right),
$$

and we write $E_{0}=E \times \times_{\mathbb{A}^{m}}\left(\mathbb{A}^{m-1} \times\{0\}\right)$ so

$$
\left(\mathbb{A}^{1}, d_{1}\{0\}\right) \otimes\left(\mathbb{A}^{1}, d_{2}\{0\}\right) \otimes \cdots \otimes\{0\}=\left(\mathbb{A}^{m-1} \times\{0\}, E_{0}\right) .
$$


Now we have a Cartesian cubic diagram

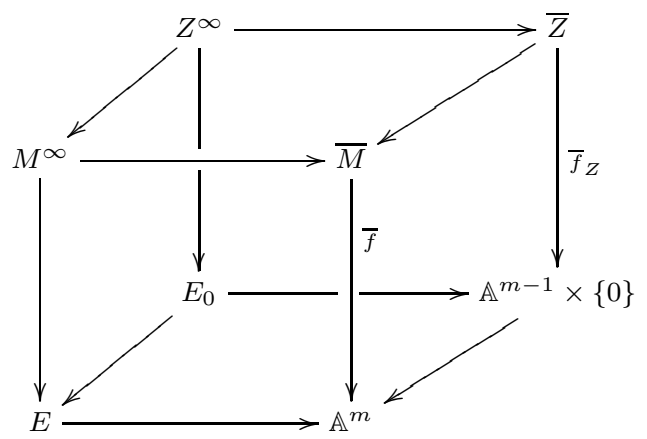

By the above diagram, we know $f_{Z}:\left(\bar{Z}, Z^{\infty}\right) \rightarrow\left(\mathbb{A}^{m-1} \times\{0\}, E_{0}\right)$ is a minimal étale map. Now we consider the fibre product,

and

$$
\bar{X}:=\bar{M} \times_{\mathbb{A}^{m}}\left(\bar{Z} \times_{\operatorname{Spec} k} \mathbb{A}^{1}\right)
$$

$$
X^{\infty}:=\bar{\pi}^{*} M^{\infty}
$$

where $\bar{\pi}$ is a canonical morphism $\bar{X} \rightarrow \bar{M}$. Now by [SV00, Theorem 4.10], we have a diagram $(\Omega)$ in $\operatorname{Sm}(k)$,

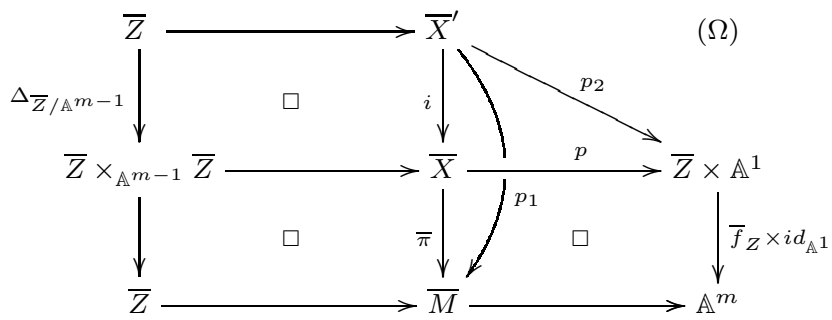

where $i: \bar{X}^{\prime} \rightarrow \bar{X}$ is an open immersion, and $p_{2}^{-1}(\bar{Z} \times\{0\})=\bar{Z}$. By Lemma $6.2, \beta\left(\mathfrak{M} / \mathfrak{M}_{\mathfrak{Z}}, c l\right)$ is an isomorphism if and only if $\beta_{c l}:\left(\underline{\mathbf{M}}\left(\left(\bar{Z}, Z^{\infty}\right) \otimes\left(\mathbb{A}^{1}, \emptyset\right)\right) / \underline{\mathbf{M}}\left(\left(\bar{Z}, Z^{\infty}\right) \otimes\left(\mathbb{A}^{1},\{0\}\right)\right)\right) \rightarrow T h\left(N_{Z} \mathbb{A}_{Z}^{1}, c l\right)$ is an isomorphism. By Lemma $5.2(2)$ this $\beta_{c l}$ is the image of $\underline{\mathbf{M}}\left(\left(\mathbb{A}^{1}, \emptyset\right) /\left(\mathbb{A}^{1},\{0\}\right)\right) \rightarrow T h\left(N_{\{0\}} \mathbb{A}^{1}, c l\right)$ under $\left(\bar{Z}, Z^{\infty}\right) \otimes-$. But this is an isomorphism by Lemma $5.2(0)$.

\section{Application}

Heuristically, the motive with modulus $M\left(\bar{X}, X^{\infty}\right)$ is a place holder which represents the cohomology of $X^{\circ}=\bar{X} \backslash X^{\infty}$ whose ramification along the support of $X^{\infty}$ is bounded by the multiplicities of $X^{\infty}$. In particular, the case when $X^{\infty}$ is reduced corresponds to tamely ramified cohomology classes. On the other hand, there are concrete connections between tame class field theory and Voevodsky's DM ${ }^{\text {eff }}$, cf., the relationship between the tame fundamental group and Suslin homology demonstrated by Geisser, Schmidt, and Speiß. In this section we show that these two points of view are compatible.

Theorem 7.1. Let $X$ be a smooth variety over $k$ which has a compactification $\bar{X}$ such that $\bar{X}$ is smooth and $|\bar{X} \backslash X|$ is a strict normal crossings divisor on $\bar{X}$. Then the unit

$$
\underline{\mathbf{M}}\left(\bar{X},|\bar{X} \backslash X|_{\mathrm{red}}\right) \rightarrow \underline{\omega}^{\mathrm{eff}}(\mathbf{M}(X))
$$

of the adjunction $\underline{\omega}_{\mathrm{eff}}: \underline{\mathbf{M D M}} \mathbf{M}^{\mathrm{eff}} \rightleftarrows \mathbf{D M}^{\mathrm{eff}}: \underline{\omega}^{\mathrm{eff}}$ is an isomorphism.

Lemma 7.2. The functor $\underline{\omega}_{\text {eff }}$ sends the tame Gysin map $g_{3} \mathfrak{M}$ to Gysin map $g_{Z^{\circ}} M^{\circ}$ of [Voe00c, Thm.3.5.4].

Proof of Lemma 7.2. By using excision [Voe00a, Proposition 5.18], Voevodsky's construction of the Gysin map [Voe00b] can be restated in terms of the deformation space obtained by blowing up $Z^{\circ} \times\{0\}$ in $X^{\circ} \times \mathbb{P}^{1}$. The definition of the tame Gysin map is given only by geometrical morphisms, our construction corresponds to Voevodsky's construction under the functor $\underline{\omega}_{\text {eff }}$. 
Proof of Theorem 7.1. Take

$$
|\bar{X} \backslash X|_{\text {red }}=\sum_{i=1}^{n} V_{i}
$$

where each $V_{i}$ is an smooth effective Cartier divisor. We prove the claim by induction on $n$.

Let us suppose $n=1$, and write $V$ for $|\bar{X} \backslash X|_{\text {red }}=V_{1}$. We have the Gysin triangle in $\mathbf{D} \mathbf{M}^{\text {eff }}$ for the closed immersion $V \hookrightarrow \bar{X}$,

$$
\mathbf{M}(\bar{X} \backslash V) \rightarrow \mathbf{M}(\bar{X}) \stackrel{g_{V} \bar{X}}{\longrightarrow} \mathbf{M}(V)(1)[2] \stackrel{+}{\rightarrow} \mathbf{M}(\bar{X} \backslash V)[1] .
$$

Since the unit Id $\rightarrow \underline{\omega}^{\text {eff }} \underline{\omega}_{\text {eff }}$ is a natural transformation, we get a morphism of distinguished triangles

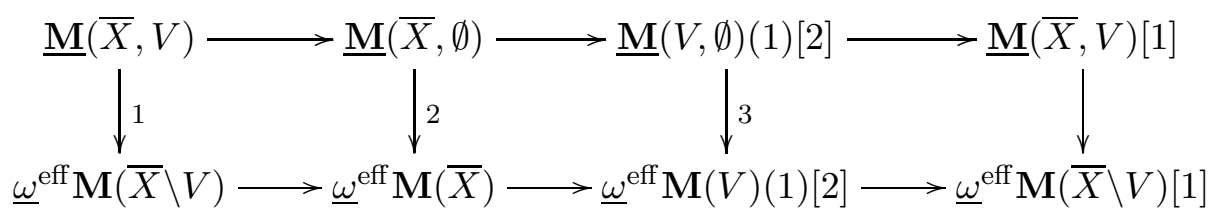

where the vertical arrows are the unit morphisms. Since $\bar{X}$ and $V$ are proper smooth over $k,(2)$ and (3) are isomorphisms. Cf. [KMSY20, Theorem6.3.1] . So (1) is also an isomorphism.

Now we take

$$
U=\bar{X} \backslash \bigcup_{i=1}^{n-1} V_{i}
$$

and

$$
W=V_{n} \backslash\left(\bigcup_{i=1}^{n-1} V_{n} \cap V_{i}\right) .
$$

It is easy to see that $U \backslash W=\bar{X} \backslash \bigcup_{i=1}^{n} V_{i}$. Now the divisor $\sum_{i=1}^{n} V_{i}$ is a strict normal crossings divisor, so $V_{n} \cdot \bar{X} V_{i}=\left|V_{n} \cap V_{i}\right|_{\text {red }}$. So we get

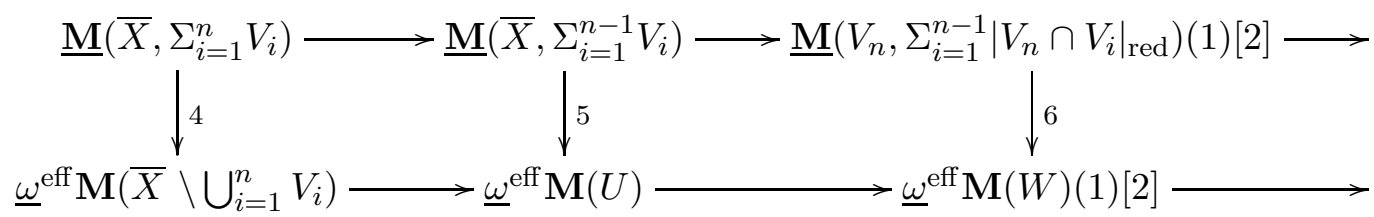

By induction, (5) and (6) are isomorphisms. So the claim is proved.

\section{The CASE of $\mathbb{Z}[1 / p]$-COEFFiCIEnTS}

In this section, we suppose that the base field has characteristic $p$. The main objective of this section is to show that the non-Voevodsky part of $\mathbf{M D M}^{\text {eff }}$ is all $p^{\infty}$-torsion in the sense that the kernel of $\omega_{\text {eff }}: \mathbf{M D M}^{\text {eff }} \rightarrow \mathbf{D M}^{\text {eff }}$ is contained in the kernel of $\mathbf{M D M}{ }^{\text {eff }} \rightarrow \mathbf{M D M}^{\text {eff }}[1 / p]$.

For a natural number $l \in \mathbb{N}$ and an integer $n \in \mathbb{Z}_{\geq 0}$, we define a presheaf $\mathbb{Z}[1 / p]_{\operatorname{tr}}\left(\square^{\left(l / p^{n}\right)}\right) \in$ $P S h(\underline{\mathbf{M C}} \mathbf{C o r}, \mathbb{Z}[1 / p])$ as

$$
\mathbb{Z}[1 / p]_{\operatorname{tr}}\left(\bar{\square}^{\left(l / p^{n}\right)}\right):\left(\bar{M}, M^{\infty}\right) \mapsto \underline{\operatorname{MCor}}\left(\left(\bar{M}, p^{n} M^{\infty}\right),\left(\mathbb{P}^{1}, l\{\infty\}\right)\right) \otimes \mathbb{Z}[1 / p] .
$$

Let us define morphisms

$$
\begin{gathered}
V^{(n)}: \mathbb{Z}[1 / p]_{\operatorname{tr}}\left(\bar{\square}^{\left(l / p^{n}\right)}\right) \rightarrow \mathbb{Z}[1 / p]_{\operatorname{tr}}\left(\bar{\square}^{\left(l / p^{n+1}\right)}\right), \\
\qquad F^{(n)}: \mathbb{Z}[1 / p]_{\operatorname{tr}}\left(\bar{\square}^{\left(l / p^{n+1}\right)}\right) \rightarrow \mathbb{Z}[1 / p]_{\operatorname{tr}}\left(\bar{\square}^{\left(l / p^{n}\right)}\right), \\
\text { satisfying } \quad V^{(n)} \circ F^{(n)}=p \cdot i d, \quad F^{(n)} \circ V^{(n)}=p \cdot i d .
\end{gathered}
$$

We use the morphism of modulus pairs $\tilde{\pi}:\left(\mathbb{P}^{1}, l p\{\infty\}\right) \rightarrow\left(\mathbb{P}^{1}, l\{\infty\}\right)$ defined by $k[x] \leftarrow k[x] ; x^{p} \leftarrow x$. Note, this is a minimal morphism which is finite flat on the total space, and therefore has a well defined transpose $\tilde{\pi}^{\mathrm{t}}:\left(\mathbb{P}^{1}, l\{\infty\}\right) \rightarrow\left(\mathbb{P}^{1}, l p\{\infty\}\right)$ as follows. 
Lemma 8.1. For a minimal finite flat morphism $g:\left(\bar{X}, X^{\infty}\right) \rightarrow\left(\bar{Y}, Y^{\infty}\right)$, we denote by $g^{\circ}$ the morphism $\bar{X} \backslash X^{\infty} \rightarrow \bar{Y} \backslash Y^{\infty}$ given arise to $g$. Then the transpose correspondence $g^{\circ t} \in \operatorname{Cor}\left(\bar{Y} \backslash Y^{\infty}, \bar{X} \backslash X^{\infty}\right)$ lies in the subgroup $\underline{\operatorname{M}} \mathbf{C o r}\left(\left(\bar{Y}, Y^{\infty}\right),\left(\bar{X}, X^{\infty}\right)\right)$.

We write $g^{t}$ for $g^{\circ t}$ considered as a morphism of modulus pairs.

Proof. It's left proper because $g$ is finite, and admissible because $g$ is minimal.

Definition 8.2. For a modulus pair $\left(\bar{M}, M^{\infty}\right)$, we define $V^{(n)}\left(\bar{M}, M^{\infty}\right)$ as the morphism given by

$$
\begin{aligned}
\underline{\operatorname{MCor}}\left(\left(\bar{M}, p^{n} M^{\infty}\right),\left(\mathbb{P}^{1}, l\{\infty\}\right)\right) & =\underline{\operatorname{MCor}}\left(\left(\bar{M}, p^{n+1} M^{\infty}\right),\left(\mathbb{P}^{1}, l p\{\infty\}\right)\right) \\
& \stackrel{\tilde{\pi} \circ-}{\longrightarrow} \underline{\operatorname{MCor}}\left(\left(\bar{M}, p^{n+1} M^{\infty}\right),\left(\mathbb{P}^{1}, l\{\infty\}\right)\right)
\end{aligned}
$$

and $F^{(n)}\left(\bar{M}, M^{\infty}\right)$ as the morphism given by

$$
\begin{aligned}
\underline{\operatorname{MCor}}\left(\left(\bar{M}, p^{n+1} M^{\infty}\right),\left(\mathbb{P}^{1}, l\{\infty\}\right)\right) \stackrel{\tilde{\pi}^{\mathrm{t} o-}}{\longrightarrow} & \underline{\operatorname{MCor}}\left(\left(\bar{M}, p^{n+1} M^{\infty}\right),\left(\mathbb{P}^{1}, l p\{\infty\}\right)\right) \\
= & \underline{\operatorname{MCor}}\left(\left(\bar{M}, p^{n} M^{\infty}\right),\left(\mathbb{P}^{1}, l\{\infty\}\right)\right)
\end{aligned}
$$

Lemma 8.3. $V^{(n)} \circ F^{(n)}=p \cdot i d$ and $F^{(n)} \circ V^{(n)}=p \cdot i d$.

Proof. We write $\pi$ for the morphism $\mathbb{A}^{1} \rightarrow \mathbb{A}^{1}$ given by the morphism of $k$-algebras $k[x] \leftarrow k[x] ; x^{p} \leftarrow x$. To prove the claim, it is enough to prove that $\pi \circ \pi^{t}=p \cdot i d \in \operatorname{Cor}\left(\mathbb{A}^{1}, \mathbb{A}^{1}\right)$ and $\pi^{t} \circ \pi=p \cdot i d \in \operatorname{Cor}\left(\mathbb{A}^{1}, \mathbb{A}^{1}\right)$. Since $\pi$ is a flat, finite, surjective morphism with degree $p$, it follows that $\pi \circ \pi^{t}=p \cdot i d \in \operatorname{Cor}\left(\mathbb{A}^{1}, \mathbb{A}^{1}\right)$ is true. So the problem is the other equality.

We need the following lemma.

Lemma 8.4. The flat pull back $(i d \times \pi)^{*}: Z^{1}\left(\mathbb{A}^{1} \times \mathbb{A}^{1}\right) \rightarrow Z^{1}\left(\mathbb{A}^{1} \times \mathbb{A}^{1}\right)$ sends $\Gamma_{\pi}$ to $p \cdot i d$. Where $\Gamma_{\pi}$ is the graph of $\pi$, i.e., $\Gamma_{\pi}: \mathbb{A}^{1} \rightarrow \mathbb{A}^{1} \times \mathbb{A}^{1} ; a \mapsto\left(a, a^{p}\right)$.

Proof of Lemma 8.4. The ideal of $k[x] \otimes_{k} k[y]$ corresponding to $\Gamma_{\pi}$ is $\left(x^{p}-y\right)$. Now id $\times \pi$ comes from the $k$-morphism $k[x] \otimes_{k} k[y] \rightarrow k[x] \otimes_{k} k[y] ; x \mapsto x, y \mapsto y^{p}$. So the pullback of the ideal sheaf $(\mathrm{id} \times \pi)^{*}\left(\left(x^{p}-y\right)\right)$ is the ideal sheaf $\left(x^{p}-y^{p}\right)$, But $\operatorname{ch}(k)=p$, so this is equal to $(x-y)^{p}$. The ideal $(x-y)$ is corresponds to the diagonal morphism $\Delta_{\mathbb{A}^{1}}$, i.e., the identity morphism in in $\operatorname{Cor}\left(\mathbb{A}^{1}, \mathbb{A}^{1}\right)$.

Now we recall $\pi$ and $\pi^{t}$ in $\operatorname{Cor}\left(\mathbb{A}^{1}, \mathbb{A}^{1}\right)$. The map $\pi$ is the graph map $\Gamma_{\pi}: \mathbb{A}^{1} \rightarrow \mathbb{A}^{1} \times \mathbb{A}^{1} ; a \mapsto\left(a, a^{p}\right)$, and $\pi^{t}$ is the map $\psi: \mathbb{A}^{1} \rightarrow \mathbb{A}^{1} \times \mathbb{A}^{1} ; b \rightarrow\left(b^{p}, b\right)$. We recall the composition $\pi^{t} \circ \pi$, it is

$$
\pi^{t} \circ \pi=p_{13 *}\left(\left(\Gamma_{\pi} \times \mathbb{A}^{1}\right) \cdot \mathbb{A}^{1} \times \mathbb{A}^{1} \times \mathbb{A}^{1}\left(\mathbb{A}^{1} \times \psi\right)\right) .
$$

Now $\Gamma_{\pi} \times \mathbb{A}^{1}$ and $\mathbb{A}^{1} \times \psi$ are effective Cartier divisors, and they are intersect properly, so

$$
\left(\Gamma_{\pi} \times \mathbb{A}^{1}\right) \times_{\mathbb{A}^{1} \times \mathbb{A}^{1} \times \mathbb{A}^{1}}\left(\mathbb{A}^{1} \times \psi\right)=\left(\Gamma_{\pi} \times \mathbb{A}^{1}\right) \cdot \mathbb{A}^{1} \times \mathbb{A}^{1} \times \mathbb{A}^{1}\left(\mathbb{A}^{1} \times \psi\right) .
$$

Now this is denoted by $V$. Then we have following diagram

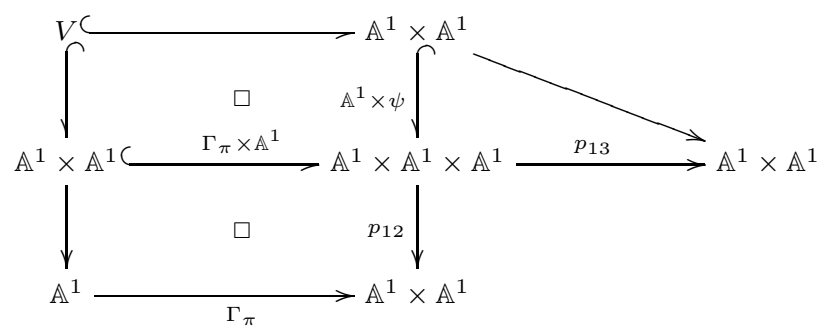

By definition, we get

$$
p_{13} \circ\left(\mathbb{A}^{1} \times \psi\right)=\operatorname{id}_{\mathbb{A}^{1} \times \mathbb{A}^{1}}
$$

and

$$
p_{12} \circ\left(\mathbb{A}^{1} \times \psi\right)=\operatorname{id}_{\mathbb{A}^{1}} \times \pi .
$$


The equality (8.2) claims that $V$ is the flat pull back $\left(i d_{\mathbb{A}^{1}} \times \pi\right)^{*}\left(\Gamma_{\pi}\right)$, by Lemma 8.4 and [Ful98, Propostion 7.1 ] we get

$$
V=p \cdot \mathrm{id}
$$

By (8.1) we get that $\pi^{t} \circ \pi=p_{13 *}(V)=V$. Therefore $\pi^{t} \circ \pi=p \cdot \operatorname{id} \operatorname{in} \operatorname{Cor}\left(\mathbb{A}^{1}, \mathbb{A}^{1}\right)$.

We consider two colimits $\underline{\lim }_{n}\left(\mathbb{Z}[1 / p]_{\operatorname{tr}}\left(\bar{\square}^{\left(l / p^{n}\right)}\right), V^{(n)}\right)$ and $\underline{\lim }_{n}\left(\underline{\omega}^{*} \mathbb{Z}[1 / p]_{\operatorname{tr}}\left(\mathbb{A}^{1}\right), \underline{\omega}^{*} \pi\right)$ in the category $P S h\left(\underline{\text { MCor })}\right.$ where the transition maps are $V^{(n)}: \mathbb{Z}[1 / p]_{\operatorname{tr}}\left(\bar{\square}^{\left(l / p^{n}\right)}\right) \rightarrow \mathbb{Z}[1 / p]_{\operatorname{tr}}\left(\bar{\square}^{\left(l / p^{n+1}\right)}\right)$ and $\underline{\omega}^{*} \pi:$ $\underline{\omega}^{*} \mathbb{Z}[1 / p]_{\operatorname{tr}}\left(\mathbb{A}^{1}\right) \rightarrow \underline{\omega}^{*} \mathbb{Z}[1 / p]_{\operatorname{tr}}\left(\mathbb{A}^{1}\right)$, and the morphism

$$
I: \underset{n}{\lim }\left(\mathbb{Z}[1 / p]_{\operatorname{tr}}\left(\bar{\square}^{\left(l / p^{n}\right)}\right), V^{(n)}\right) \rightarrow \underset{n}{\lim }\left(\underline{\omega}^{*} \mathbb{Z}[1 / p]_{\operatorname{tr}}\left(\mathbb{A}^{1}\right), \underline{\omega}^{*} \pi\right)
$$

given by the natural immersions $\mathbb{Z}[1 / p]_{\operatorname{tr}}\left(\bar{\square}^{\left(l / p^{n}\right)}\right) \rightarrow \underline{\omega}^{*} \mathbb{Z}[1 / p]_{\operatorname{tr}}\left(\mathbb{A}^{1}\right)$.

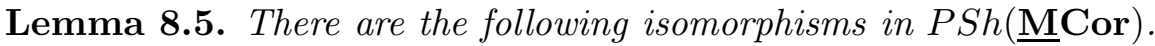

$$
\begin{aligned}
\mathbb{Z}[1 / p]_{\operatorname{tr}}\left(\bar{\square}^{(l)}\right) & \simeq \underset{n}{\lim }\left(\mathbb{Z}[1 / p]_{\operatorname{tr}}\left(\bar{\square}^{\left(l / p^{n}\right)}\right), V^{(n)}\right) \\
\underline{\omega}^{*} \mathbb{Z}[1 / p]_{\operatorname{tr}}\left(\mathbb{A}^{1}\right) & \simeq \underset{n}{\lim }\left(\underline{\omega}^{*} \mathbb{Z}[1 / p]_{t r}\left(\mathbb{A}^{1}\right), \underline{\omega}^{*} \pi\right)
\end{aligned}
$$

Proof. The prime $p$ is invertible in $\mathbb{Z}[1 / p]$ so by Lemma 8.3 , morphisms $V^{(n)}$ are isomorphisms. Similarly the morphism $\pi: \mathbb{Z}[1 / p]_{\operatorname{tr}}\left(\mathbb{A}^{1}\right) \rightarrow \mathbb{Z}[1 / p]_{\operatorname{tr}}\left(\mathbb{A}^{1}\right)$ is an isomorphism. So the claim follows.

Lemma 8.6. $I$ is an isomorphism.

Proof. The problem is surjectivity. By [KMSY19a, Lemma 1.1.3]

$$
\operatorname{Cor}\left(\bar{M} \backslash M^{\infty}, \mathbb{A}^{1}\right)=\bigcup_{n} \underline{\operatorname{MC}} \operatorname{Cor}\left(\left(\bar{M}, p^{n} M^{\infty}\right),\left(\mathbb{P}^{1}, l\{\infty\}\right)\right) .
$$

Hence, for any elementary correspondence $W \in \operatorname{Cor}\left(\bar{M} \backslash M^{\infty}, \mathbb{A}^{1}\right)$, there is an integer $n$ such that $W \in$ $\underline{\operatorname{MCor}}\left(\left(\bar{M}, p^{n} M^{\infty}\right),\left(\mathbb{P}^{1}, l\{\infty\}\right)\right)$.

This lemma implies the following theorem which only holds in positive characteristic.

Theorem 8.7. For any $l \in \mathbb{Z}_{\geq 1}, \underline{\mathbf{M}}\left(\left(\mathbb{P}^{1},\{\infty\}\right) /\left(\mathbb{P}^{1}, l\{\infty\}\right)\right) \otimes \mathbb{Z}[1 / p]=0$.

Proof. Since $\mathbb{Z}[1 / p]$ is a flat $\mathbb{Z}$-module, it is enough to show that the natural morphism $\mathbb{Z}[1 / p]_{\operatorname{tr}}\left(\mathbb{P}^{1}, l\{\infty\}\right) \rightarrow$ $\mathbb{Z}[1 / p]_{\operatorname{tr}}\left(\mathbb{P}^{1},\{\infty\}\right)$ is an isomorphism. There is a commutative diagram

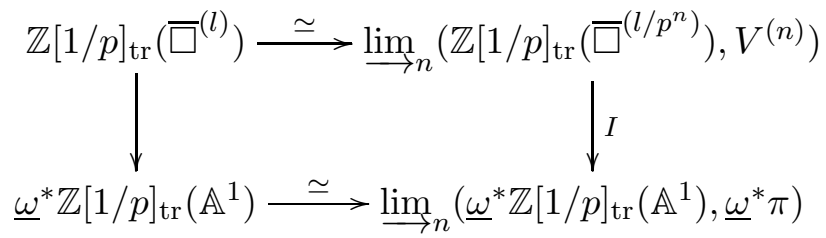

in $P S h(\underline{\mathbf{M C o r}})$, where vertical maps are natural inclusions and horizontal maps are isomophisms given by Lemma 8.5. By Lemma 8.6 we know that $I$ is an isomorphism. So the natural inclusion $\mathbb{Z}[1 / p]_{\operatorname{tr}}\left(\bar{\square}^{(l)}\right) \rightarrow$ $\underline{\omega}^{*} \mathbb{Z}[1 / p]_{\operatorname{tr}}\left(\mathbb{A}^{1}\right)$ is also an isomophism for all $l \geq 1$. The result now follows from the sequence of inclusions $\mathbb{Z}[1 / p]_{\operatorname{tr}}\left(\bar{\square}^{(l)}\right) \hookrightarrow \mathbb{Z}[1 / p]_{\operatorname{tr}}(\bar{\square}) \hookrightarrow \underline{\omega}^{*} \mathbb{Z}[1 / p]_{\operatorname{tr}}\left(\mathbb{A}^{1}\right)$.

Corollary 8.8. For any modulus pair $\left(\bar{M}, M^{\infty}\right)$ such that $\bar{M}$ is smooth and $M_{\text {red }}^{\infty}$ is strict normal crossing,

$$
\underline{\mathbf{M}}\left(\bar{M}, M^{\infty}\right) \otimes \mathbb{Z}[1 / p] \simeq \underline{\mathbf{M}}\left(\bar{M}, M_{\text {red }}^{\infty}\right) \otimes \mathbb{Z}[1 / p] .
$$

Proof. Set $M^{\infty}=\sum_{k=1}^{n} n_{k} V_{k}$ where $V_{k}$ are smooth Cartier divisor. We take $M_{i}^{\infty}:=n_{1} V_{1}+\cdots n_{i} V_{i}+$ $\sum_{k=i+1}^{n} V_{k}$, it is enough to prove $\underline{\mathbf{M}}\left(\bar{M}, M_{i}^{\infty}\right) \otimes \mathbb{Z}[1 / p] \simeq \underline{\mathbf{M}}\left(\bar{M}, M_{i-1}^{\infty}\right) \otimes \mathbb{Z}[1 / p]$. By Mayer-Vietoris sequence, we can replace $\underline{\mathbf{M}}\left(\bar{M}, M_{i}^{\infty}\right) \otimes \mathbb{Z}[1 / p]$ by $\underline{\mathbf{M}}\left(\bar{U}, \bar{U} \cap M_{i}^{\infty}\right) \otimes \mathbb{Z}[1 / p]$ where $\bar{U}$ has a local chart $q: \bar{U} \rightarrow \mathbb{A}^{m}$ such that $\bar{U} \cap V_{i}=q^{-1}\left(\mathbb{A}^{m-1} \times\{0\}\right)$ and $\bar{U} \cap\left(M_{i}^{\infty}-n_{i} V_{i}\right)=q^{-1}\left(\left\{T_{1}^{d_{1}} \ldots . . T_{j}^{d_{s}}=0\right\}\right)$ where 
$T_{l}$ are the coordinates of $\mathbb{A}^{m}$. Replace $\underline{\mathbf{M}}\left(\bar{M}, M_{i}^{\infty}\right) \otimes \mathbb{Z}[1 / p]$ by $\underline{\mathbf{M}}\left(\bar{U}, \bar{U} \cap M_{i}^{\infty}\right) \otimes \mathbb{Z}[1 / p]$. In this case we have a diagram $(\Omega)$ used in the proof of the tame Gysin triangle. By Proposition 3.1 the cone of the natural morphisms $\underline{\mathbf{M}}\left(\bar{M}, M_{i}^{\infty}\right) \otimes \mathbb{Z}[1 / p] \rightarrow \underline{\mathbf{M}}\left(\bar{M}, M_{i-1}^{\infty}\right) \otimes \mathbb{Z}[1 / p]$ is isomorphic to $\underline{\mathbf{M}}\left(\left(V_{i}, V_{i}^{\infty}\right) \otimes\right.$ $\left.\left(\mathbb{A}^{1},\{0\}\right)\right) / \underline{\mathbf{M}}\left(\left(V_{i}, V_{i}^{\infty}\right) \otimes\left(\mathbb{A}^{1}, n_{i}\{0\}\right)\right) \otimes \mathbb{Z}[1 / p]$ where $V_{i}^{\infty}=V_{i} \cdot \bar{M}\left(n_{1} V_{1}+\cdots n_{i-1} V_{i-1}+\Sigma_{k=i+1}^{n} V_{k}\right)$, Proposition 3.1 and Theorem 8.7 claims $\left(\mathbb{A}^{1},\{0\}\right) /\left(\mathbb{A}^{1}, n_{i}\{0\}\right) \otimes \mathbb{Z}[1 / p]=0$ we win.

By this corollary and Theorem 7.1, we get the following theorem.

Theorem 8.9 (Corollary 1.6). If the base field $k$ has characteristic $p$ and admits log resolution of singularities, then there is an equivalence

$$
\omega_{\text {eff }}[1 / p]: \mathbf{M D M}^{\mathrm{eff}}[1 / p] \stackrel{\cong}{\longrightarrow} \mathbf{D M}^{\mathrm{eff}}[1 / p]
$$

Proof. We omit $[1 / p]$. Since we assume the base field $k$ admits log resolution of singularities, any modulus pair is isomorphic to a modulus pair which has a smooth total space and strictly normal crossing divisor modulus. Now $\mathbf{M D M} \mathbf{M}^{\text {eff }}$ is compactly generated by the $\underline{\mathbf{M}}\left(\bar{M}, M^{\infty}\right)$, [KMSY19b, Theorem $\left.1(2)\right]$, and both $\omega_{\text {eff }}$ and $\omega^{\text {eff }}$ commute with all sums (the latter because $\omega_{\text {eff }}$ sends compact generators to compact objects), so it suffices to know that $\underline{\mathbf{M}}\left(\bar{M}, M^{\infty}\right) \rightarrow \omega_{\text {eff }} \omega^{\text {eff }} \underline{\mathbf{M}}\left(\bar{M}, M^{\infty}\right)$ is an isomorphism when $\bar{M}$ is smooth and proper and $M^{\infty}$ is a strict normal crossings divisor. If $M^{\infty}$ is reduced, Theorem 7.1 implies the claim. By Corollary 8.8 its also true when $M^{\infty}$ is not reduced.

\section{REFERENCES}

[AGV71] Michael Artin, Alexander Grothendieck, and Jean-Louis Verdier. Théorie de Topos et Cohomologie Étale des Schémas I, II, III, volume 269, 270, 305 of Lecture Notes in Mathematics. Springer, 1971.

[Ayo14] Joseph Ayoub. La réalisation étale et les opérations de Grothendieck. Ann. Sci. Éc. Norm. Supér. (4), 47(1):1$145,2014$.

[BCKS17] F. Binda, J. Cao, W. Kai, and R. Sugiyama. Torsion and divisibility for reciprocity sheaves and 0-cycles with modulus. J. Algebra, 469:437-463, 2017.

[BE03] Spencer Bloch and Hélène Esnault. The additive dilogarithm. Number Extra Vol., pages 131-155. 2003. Kazuya Kato's fiftieth birthday.

[Bes00] Amnon Besser. Syntomic regulators and p-adic integration. I. Rigid syntomic regulators. In Proceedings of the Conference on p-adic Aspects of the Theory of Automorphic Representations (Jerusalem, 1998), volume 120, pages 291-334, 2000.

[BM20] Federico Binda and Alberto Merici. Connectivity and purity for logarithmic motives. arXiv:2012.08361, 2020.

[BPØ20] Federico Binda, Doosung Park, and Paul Arne Østvær. Triangulated categories of logarithmic motives over a field. arXiv:2004.12298, 2020.

[BS18] Federico Binda and Shuji Saito. Semi-purity for cycles with modulus. arXiv:1812.01878, 2018.

[BS19] Federico Binda and Shuji Saito. Relative cycles with moduli and regulator maps. J. Inst. Math. Jussieu, 18(6):1233-1293, 2019.

[CT03] Bruno Chiarellotto and Nobuo Tsuzuki. Cohomological descent of rigid cohomology for étale coverings. Rend. Sem. Mat. Univ. Padova, 109:63-215, 2003.

[Ful98] William Fulton. Intersection theory, volume 2 of Ergebnisse der Mathematik und ihrer Grenzgebiete. 3. Folge. A Series of Modern Surveys in Mathematics [Results in Mathematics and Related Areas. 3rd Series. A Series of Modern Surveys in Mathematics]. Springer-Verlag, Berlin, second edition, 1998.

[KMSY19a] Bruno Kahn, Hiroyasu Miyazaki, Shuji Saito, and Takao Yamazaki. Motives with modulus, I: Modulus sheaves with transfers for non-proper modulus pairs. arXiv:1908.02975, 2019.

[KMSY19b] Bruno Kahn, Hiroyasu Miyazaki, Shuji Saito, and Takao Yamazaki. Motives with modulus, II: Modulus sheaves with transfers for proper modulus pairs. arXiv:1910.14534, 2019.

[KMSY20] Bruno Kahn, Hiroyasu Miyazaki, Shuji Saito, and Takao Yamazaki. Motives with modulus, III: The categories of motives. arXiv:2011.11859, 2020.

[Koi] Jun Koizumi. private communication.

[KS19] Shane Kelly and Shuji Saito. Smooth blowup square for motives with modulus. arXiv:1907.12759, 2019.

[KSY14] Bruno Kahn, Shuji Saito, and Takao Yamazaki. Reciprocity sheaves. 2014.

[KSY17] Bruno Kahn, Shuji Saito, and Takao Yamazaki. Reciprocity sheaves, II. arXiv:1707.07398, 2017.

[Miy19] Hiroyasu Miyazaki. Cube invariance of higher Chow groups with modulus. J. Algebraic Geom., 28(2):339-390, 2019.

[MR09] James S. Milne and Niranjan Ramachandran. Motivic complexes over finite fields and the ring of correspondences at the generic point. Pure Appl. Math. Q., 5(4, Special Issue: In honor of John Tate. Part 1):1219-1252, 2009. 
[NS08] Yukiyoshi Nakkajima and Atsushi Shiho. Weight filtrations on log crystalline cohomologies of families of open smooth varieties, volume 1959 of Lecture Notes in Mathematics. Springer-Verlag, Berlin, 2008.

[Shi02] Atsushi Shiho. Crystalline fundamental groups. II. Log convergent cohomology and rigid cohomology. J. Math. Sci. Univ. Tokyo, 9(1):1-163, 2002.

[SV00] Andrei Suslin and Vladimir Voevodsky. Bloch-Kato conjecture and motivic cohomology with finite coefficients. In The arithmetic and geometry of algebraic cycles (Banff, AB, 1998), volume 548 of NATO Sci. Ser. C Math. Phys. Sci., pages 117-189. Kluwer Acad. Publ., Dordrecht, 2000.

[Voe00a] Vladimir Voevodsky. Cohomological theory of presheaves with transfers, volume 143 of Ann. of Math. Stud., page 87-137. Princeton Univ. Press, Princeton, NJ, 2000.

[Voe00b] Vladimir Voevodsky. Cohomological theory of presheaves with transfers. In Cycles, transfers, and motivic homology theories, volume 143 of Ann. of Math. Stud., pages 87-137. Princeton Univ. Press, Princeton, NJ, 2000.

[Voe00c] Vladimir Voevodsky. Triangulated categories of motives over a field. In Cycles, transfers, and motivic homology theories, volume 143 of Ann. of Math. Stud., pages 188-238. Princeton Univ. Press, Princeton, NJ, 2000. 